\title{
CATEGORICAL ABSTRACT ALGEBRAIC LOGIC: TARSKI CONGRUENCE SYSTEMS, LOGICAL MORPHISMS AND LOGICAL QUOTIENTS
}

\section{GEORGE VOUTSADAKIS}

School of Mathematics and Computer Science

Lake Superior State University

Sault Sainte Marie

MI 49783

USA

e-mail: gvoutsad@lssu.edu

\begin{abstract}
A general notion of a congruence system is introduced for $\pi$-institutions. Congruence systems in this sense are collections of equivalence relations on the sets of sentences of the $\pi$-institution that are preserved both by signature morphisms and by fixed collections of natural transformations from finite tuples of sentences to sentences. Based on this notion of a congruence system, the notion of a Tarski congruence system, generalizing the notion of a Tarski congruence from sentential logics, is considered. Logical and bilogical morphisms are introduced for $\pi$-institutions, also generalizing similar concepts from the theory of sentential logics, and their relationship with the familiar translations and interpretations of institutions is discussed. Finally, the interplay between these logical maps and the formation of logical quotients of $\pi$-institutions and the way they transform the Tarski congruence systems is investigated.
\end{abstract}

2010 Mathematics Subject Classification: 03G27.

Keywords and phrases: abstract algebraic logic, deductive systems, institutions, equivalent deductive systems, algebraizable deductive systems, adjunctions, equivalent institutions, algebraizable institutions, Leibniz congruence, Tarski congruence, algebraizable sentential logics.

Received March 23, 2015

(C) 2015 Scientific Advances Publishers 


\section{Introduction}

In [4], Blok and Pigozzi introduced the concept of the Leibniz congruence associated with the theories of a deductive system. Leibniz congruences are, more generally, associated with filters of logical matrices; the case of theories, i.e., filters on formula algebras, being a special case. More specifically, given a logical matrix $\mathfrak{A}=\langle\mathbf{A}, F\rangle$, the Leibniz congruence associated with $\mathfrak{A}$ is the largest congruence on the algebra A that is compatible with $F$, in the sense that $F$ is the union of equivalence classes of the congruence. Properties of the Leibniz congruence give rise to the abstract algebraic hierarchy of logics, consisting of the major classes of protoalgebraic [3], equivalential [7], and algebraizable [4] logics (see also [12] for an excellent overview). In subsequent work, Font and Jansana [11] generalized the work of Blok and Pigozzi by considering the notion of a Tarski congruence of an abstract logic. An abstract logic $\mathbb{L}=\langle\mathbf{A}, C\rangle$ consists of an algebra $\mathbf{A}$ together with a closure operator $C$ on $A$, the universe of $\mathbf{A}$. The Tarski congruence associated with the abstract logic $\mathbb{L}$ is the largest congruence that is compatible with all closed sets of the closure operator $C$. Both the Leibniz and the Tarksi congruence of a logic provide significant tools for the investigation of the algebraizability of a logic and for the study of the connections between metalogical properties of logics and corresponding algebraic properties. Except for [11] and [12], [8] and the very recent [1] are other excellent expositions of the rôle that congruences with compatibility properties play in studying the interaction between logical and algebraic properties.

Despite the successful generalization of the algebraizability framework to a categorical, more abstract, level, initially by the author in [22, 21] and more recently by the work of a group of colleagues [15, 13, 14], able to cover the case of an institutional logic, there had not been any notion of "congruence" pertaining to $\pi$-institutions, which would allow, at least partially, carrying some of the universal algebraic results to this level. In 
the present paper, we introduce an abstract notion of a congruence system, which consists of a collection of equivalence relations on the sentences of a $\pi$-institution that are preserved by both signature morphisms and selected classes of finitary natural transformations from sentences to sentences. It coincides with the usual universal algebraic notion of congruence in some special $\pi$-institutions but is different, in general. Based on this notion, the notion of a Tarksi congruence system is defined for this framework. Roughly speaking, a Tarski congruence system is a congruence system in this new sense whose component over a given signature is compatible with every theory over that signature. This definition follows the definition of the Tarski congruences of Font and Jansana [11]. Tarski congruence systems are studied in the first part of the paper.

In the second part, the notions of a logical and of a bilogical morphism of the sentential logic framework (see [11]) are adapted to the $\pi$-institution level. Roughly speaking, a logical morphism is an algebra homomorphism that preserves the logical closure structure in the forward direction, whereas a bilogical morphism is a surjective algebra homomorphism that preserves the logical structure both in the forward and the backward direction. As a result, a bilogical morphism between two abstract logics induces an isomorphism between the two closure structures. The adapted notions share similar properties and it is shown that, subject to having isomorphisms as signature functor components, the institutional bilogical morphisms also induce isomorphisms between the theory categories of the related $\pi$-institutions.

An interesting, but not so surprising, result is that both logical and bilogical morphisms are intimately connected with notions of morphisms that had previously been considered in the categorical framework [21]. These are semi-interpretations and interpretations between $\pi$-institutions.

The connections are given in detail in the second part of the paper, where a result concerning preservation of Tarski congruence systems under bilogical morphisms is also presented. 
Finally, in the third part of the paper, logical quotients of $\pi$-institutions by logical congruence systems are constructed. Many of the correspondence results between surjective homomorphisms and congruences that carry on from universal algebra into abstract logics in the form of correspondences between logical morphisms and logical quotients [11] are now lifted to the $\pi$-institution level.

The reader is referred to either of [2, 5, 20] for all unexplained categorical notation, to $[16,17]$ for the introduction and the basic concepts pertaining to institutions and to [10] for those on $\pi$-institutions, and, finally, to [21] for the introduction of translations and interpretations between $\pi$-institutions. In [19], a comparison is given of many of the different notions of morphisms that have been introduced in the theory of institutions, some of which are related to the ones used here.

\section{Sentential Logics and $\pi$-Institutions}

In this section, bits of the theory of sentential logics, that serves as the paradigm for the present theory and may be viewed as the primary motivation for its development, are presented. Discussing these aspects from the theory of sentential logics and the theory of $\pi$-institutions will also facilitate the understanding of the notions and the results developed in later sections, where references and comparisons with these two theories will frequently be made. The primary reference sources for the material on sentential logics from the point of view of abstract algebraic logic are $[11,4,8]$.

Recall that a logical matrix $\mathfrak{A}=\langle\mathbf{A}, F\rangle$ is a pair consisting of an algebra $\mathbf{A}=\left\langle A, \mathcal{L}^{\mathbf{A}}\right\rangle$ and a subset $F \subseteq A$, called the filter of $\mathfrak{A}$. A congruence $\theta$ of $\mathbf{A}$ is said to be compatible with $F$ if $F$ is a union of $\theta$-equivalence classes, i.e., if, for all $a, b \in A,\langle a, b\rangle \in \theta$ and $a \in F$ imply $b \in F$. In this case $\theta$ is called a matrix congruence of $\mathfrak{A}$. The collection 
of all congruences of the algebra $\mathbf{A}$ forms a complete lattice under inclusion. The collection of all matrix congruences of $\mathfrak{A}$ forms a principal ideal of this lattice and its maximum element is called the Leibniz congruence of $\mathfrak{A}$ and denoted by $\Omega(\mathfrak{A})$ or $\Omega_{\mathbf{A}}(F)$. Blok and Pigozzi [4] introduced this congruence and they proved that, for all $a, b \in A$,

$$
\begin{aligned}
\langle a, b\rangle \in \Omega_{\mathbf{A}}(F) \quad \text { iff } \quad & \forall \phi(p, \vec{q}) \in \operatorname{Fm}_{\mathcal{L}} \forall \vec{c} \in A^{k}, \\
& \phi^{\mathbf{A}}(a, \vec{c}) \in F \Leftrightarrow \phi^{\mathbf{A}}(b, \vec{c}) \in F,
\end{aligned}
$$

where, by $\mathrm{Fm}_{\mathcal{L}}$ is denoted the set of $\mathcal{L}$-formulas in a fixed denumerable set of variables $V$ and $k$ is the length of the variable vector (of all variables different from $p$ appearing in $\phi) \vec{q}$. Extensive study of the properties of $\Omega_{\mathrm{A}}$, viewed as an operator from the lattice of filters of $\mathbf{A}$ to the lattice of congruences of $\mathbf{A}$, has given rise to an algebraic hierarchy of logics, which constitutes the backbone of the area of abstract algebraic logic. (A very good reference is [8], where the interested reader may find, apart from a description of the most important classes of this hierarchy, many more references to original works. Also, [12] provides a brief overview of the area and [1] contains a wealth of results pertaining to the so-called "operator approach" to abstract algebraic logic.)

Recall from [11] that an abstract logic $\mathbb{L}=\langle\mathbf{A}, C\rangle$ consists of an algebra $\mathbf{A}=\left\langle A, \mathcal{L}^{\mathbf{A}}\right\rangle$ together with a closure operator $C$ on $A$. In [23], an abstract logic was called a generalized matrix. A congruence $\theta$ of $A$ is said to be a logical congruence of $\mathbb{L}$, if, for all $a, b \in A$,

$$
\langle a, b\rangle \in \theta \text { implies } C(a)=C(b) .
$$

This is equivalent to $\theta$ being compatible with all $C$-closed sets of $A$. As in the case of a logical matrix, it is also the case here that the lattice of all logical congruences of $\mathbb{L}$ is a principal ideal of the complete lattice of all congruences of $\mathbf{A}$ and its largest element is called the Tarski congruence 
of $\mathbb{L}$ and denoted by $\widetilde{\Omega}(\mathbb{L})$ or $\widetilde{\Omega}_{\mathbf{A}}(C)$. The Tarski congruence of an abstract logic is the main tool of the theory developed in [11], where it is noted that the characterization of the Leibniz congruence (1) immediately yields the following characterization of the Tarski congruence:

$$
\begin{aligned}
\langle a, b\rangle \in \widetilde{\Omega}_{\mathbf{A}}(C) \quad \text { iff } \quad & \forall \phi(p, \vec{q}) \in \mathrm{Fm}_{\mathcal{L}^{\forall}} \forall \vec{c} \in A^{k}, \\
& C\left(\phi^{\mathbf{A}}(a, \vec{c})\right)=C\left(\phi^{\mathbf{A}}(b, \vec{c})\right) .
\end{aligned}
$$

The following notions of morphisms in increasing strength, introduced in [6], relate abstract logics while respecting aspects of their deductive apparatuses. Let $\mathbb{L}=\langle\mathbf{A}, C\rangle, \mathbb{L}^{\prime}=\left\langle\mathbf{A}^{\prime}, C^{\prime}\right\rangle$ be two abstract logics, where $\mathbf{A}, \mathbf{A}^{\prime}$ are similar algebras. An algebra homomorphism $h: \mathbf{A} \rightarrow \mathbf{A}^{\prime}$ is a logical morphism from $\mathbb{L}$ to $\mathbb{L}^{\prime}$ if, for every $C^{\prime}$-closed set $T^{\prime}$ of $A^{\prime}, h^{-1}\left(T^{\prime}\right)$ is a $C$-closed set of $A . \mathbb{L}$ is projectively generated from $\mathbb{L}^{\prime}$ by $h$ if $\left\{h^{-1}\left(T^{\prime}\right): T^{\prime} C^{\prime}\right.$-closed $\}$ is the entire set of $C$-closed subsets of $A$. Finally, $h$ is a bilogical morphism from $\mathbb{L}$ onto $\mathbb{L}^{\prime}$ if it is an epimorphism and projectively generates $\mathbb{L}$ from $\mathbb{L}^{\prime}$. Font and Jansana ([11], Proposition 1.5) show that an epimorphism $h: \mathbf{A} \rightarrow \mathbf{A}^{\prime}$ is a bilogical morphism from $\mathbb{L}$ onto $\mathbb{L}^{\prime}$ if and only if the lattices of $C$-closed sets of $A$ and of $C^{\prime \prime}$-closed sets of $A^{\prime}$ are isomorphic under the correspondence

$$
T \mapsto h(T), \quad T^{\prime} \mapsto h^{-1}\left(T^{\prime}\right)
$$

They also show ([11], Proposition 1.7) that, if $h: \mathbb{L} \rightarrow \mathbb{L}^{\prime}$ is a biological morphism, then

$$
\widetilde{\Omega}(\mathbb{L})=h^{-1}\left(\widetilde{\Omega}\left(\mathbb{L}^{\prime}\right)\right)
$$

Two abstract logics $\mathbb{L}$ and $\mathbb{L}^{\prime}$ are isomorphic when there is a bijective logical morphism from $\mathbb{L}$ to $\mathbb{L}^{\prime}$ whose inverse is also a logical morphism. 
Font and Jansana introduce, next, the notion of a logical quotient of an abstract logic $\mathbb{L}=\langle\mathbf{A}, C\rangle$. If $\theta$ is a congruence of $\mathbf{A}$, the closure system $\mathcal{C} / \theta=\left\{S \subseteq A / \theta: \pi_{\theta}^{-1}(S) \in \mathcal{C}\right\}$, where $\pi_{\theta}: \mathbf{A} \rightarrow \mathbf{A} / \theta$ is the natural projection and $\mathcal{C}$ is the closure system corresponding to the closure operator $C$, defines an abstract logic $\mathbb{L} / \theta=\langle\mathbf{A} / \theta, \mathcal{C} / \theta\rangle$ on the quotient algebra $\mathbf{A} / \theta$ in such a way that $\pi_{\theta}: \mathbb{L} \rightarrow \mathbb{L} / \theta$ becomes a logical morphism. If it so happens that $\theta$ is a logical congruence of $\mathbb{L}$, then $\pi_{\theta}: \mathbb{L} \rightarrow \mathbb{L} / \theta$ is a biological morphism from $\mathbb{L}$ onto $\mathbb{L} / \theta$. In Theorems 1.8-1.10 of [11], analogs of the classical homomorphism theorems of universal algebra are proved for abstract logics. It is also shown in Corollary 1.11 that, if $\theta$ is a logical congruence of an abstract logic $\mathbb{L}$, then $\widetilde{\Omega}(\mathbb{L} / \theta)=\widetilde{\Omega}(\mathbb{L}) / \theta$. Perhaps the most central notion of the theory is the notion of a reduced abstract logic. $\mathbb{L}$ is said to be reduced if $\widetilde{\Omega}(\mathbb{L})=\Delta_{A}$, i.e., if $\mathbb{L}$ has a single logical congruence. For an arbitrary abstract logic $\mathbb{L}$, its reduction $\mathbb{L}^{*}=\mathbb{L} / \widetilde{\Omega}(\mathbb{L})$ is the quotient of $\mathbb{L}$ by its Tarski congruence and it is always reduced.

Finally, before introducing the basic analogs of the theory above in the context of $\pi$-institutions, the definition of a $\pi$-institution is provided, which will be the central object of our investigations. For many more details on institutions, the reader is referred to the original sources $[16,17]$, where many examples may also be found. For $\pi$-institutions, the original reference is [10]. For other examples of logical nature, the reader may consult $[22,21]$. A lot of examples pertaining to theoretical computer science may be found in the literature, e.g., in [18, 19]. Moreover, the important rôle that institutions have played in the theory of formal specifications of data structures and programming languages, as well as their key service as the underlying structures on which a model theory that is independent of the adopted logical system may be developed, has led to the compilation of an excellent comprehensive survey [9]. 
A $\pi$-institution $\mathcal{I}=\left\langle\mathbf{S i g n}, \mathrm{SEN},\left\{C_{\sum}\right\}_{\sum \in|\operatorname{Sign}|}\right\rangle$ consists of

(i) a category Sign whose objects are called signatures;

(ii) a functor SEN : Sign $\rightarrow$ Set, from the category Sign of signatures into the category Set of sets, called the sentence functor and giving, for each signature $\Sigma$, a set whose elements are called sentences over that signature $\sum$, or $\sum$-sentences, and

(iii) a mapping $C_{\Sigma}: \mathcal{P}(\operatorname{SEN}(\Sigma)) \rightarrow \mathcal{P}(\operatorname{SEN}(\Sigma))$, for each $\Sigma \in|\operatorname{Sign}|$, called $\sum$-closure, such that

(a) $A \subseteq C_{\Sigma}(A)$, for all $\sum \in|\operatorname{Sign}|, A \subseteq \operatorname{SEN}(\Sigma)$,

(b) $C_{\Sigma}\left(C_{\Sigma}(A)\right)=C_{\Sigma}(A)$, for all $\sum \in|\operatorname{Sign}|, A \subseteq \operatorname{SEN}\left(\sum\right)$,

(c) $C_{\Sigma}(A) \subseteq C_{\Sigma}(B)$, for all $\sum \in|\operatorname{Sign}|, A \subseteq B \subseteq \operatorname{SEN}(\Sigma)$,

(d) $\operatorname{SEN}(f)\left(C_{\Sigma_{1}}(A)\right) \subseteq C_{\Sigma_{2}}(\operatorname{SEN}(f)(A))$, for all $\Sigma_{1}, \Sigma_{2} \in|\operatorname{Sign}|$, $f \in \operatorname{Sign}\left(\Sigma_{1}, \Sigma_{2}\right), A \subseteq \operatorname{SEN}\left(\Sigma_{1}\right)$.

Sometimes the focus will be on just the signature category and the sentence functor. In that case, we will suppress Sign and only speak of SEN : Sign $\rightarrow$ Set with the signature category being understood from context.

The clone of all natural transformations on SEN is the category $U$ with collection of objects $\left\{\mathrm{SEN}^{\alpha}: \alpha\right.$ an ordinal $\}$ and collection of morphisms $\tau: \mathrm{SEN}^{\alpha} \rightarrow \mathrm{SEN}^{\beta} \beta$-sequences of natural transformations $\tau: \mathrm{SEN}^{\alpha} \rightarrow \mathrm{SEN}$. Composition of $\left\langle\tau_{i}: i<\beta\right\rangle: \mathrm{SEN}^{\alpha} \rightarrow \mathrm{SEN}^{\beta}$ with $\left\langle\sigma_{j}: j<\gamma\right\rangle: \mathrm{SEN}^{\beta} \rightarrow \mathrm{SEN}^{\gamma}$

$$
\mathrm{SEN}^{\alpha} \stackrel{\left\langle\tau_{i}: i<\beta\right\rangle}{\longrightarrow} \mathrm{SEN}^{\beta} \stackrel{\left\langle\sigma_{j}: j<\gamma\right\rangle}{\longrightarrow} \mathrm{SEN}^{\gamma},
$$

is defined by

$$
\left\langle\sigma_{j}: j<\gamma\right\rangle \circ\left\langle\tau_{i}: i<\beta\right\rangle=\left\langle\sigma_{j}\left(\left\langle\tau_{i}: i<\beta\right\rangle\right): j<\gamma\right\rangle .
$$


A subcategory of this category with objects all objects of the form $\mathrm{SEN}^{k}: k<\omega$, and containing all projection morphisms $p^{k, i}: \mathrm{SEN}^{k} \rightarrow \mathrm{SEN}$, $i<k, k<\omega$, with $p_{\Sigma}^{k, i}: \operatorname{SEN}\left(\sum\right)^{k} \rightarrow \operatorname{SEN}$ given by

$$
p_{\Sigma}^{k, i}(\vec{\phi})=\phi_{i} \text {, for all } \vec{\phi} \in \operatorname{SEN}\left(\sum\right)^{k},
$$

and such that, for every family $\left\{\tau_{i}: \mathrm{SEN}^{k} \rightarrow \mathrm{SEN}: i<\ell\right\}$ of natural transformations in $N,\left\langle\tau_{i}: i<\ell\right\rangle: \mathrm{SEN}^{k} \rightarrow \mathrm{SEN}^{\ell}$ is also in $N$, is referred to as a category of natural transformations on SEN.

\section{Tarski Congruence Systems}

Let Sign be a category and SEN : Sign $\rightarrow$ Set be a functor. In addition, let $N$ be a category of natural transformations on SEN. Given $\sum \in|\operatorname{Sign}|$, an equivalence relation $\theta_{\Sigma}$ on $\operatorname{SEN}(\Sigma)$ is said to be an $N$-congruence if, for all $\sigma: \operatorname{SEN}^{k} \rightarrow \operatorname{SEN}$ in $N$ and all $\vec{\phi}, \vec{\psi} \in \operatorname{SEN}\left(\sum\right)^{k}$,

$$
\vec{\phi} \theta_{\Sigma}^{k} \vec{\psi} \quad \text { imply } \sigma_{\Sigma}(\vec{\phi}) \theta_{\Sigma} \sigma_{\Sigma}(\vec{\psi}) .
$$

Let Sign and SEN : Sign $\rightarrow$ Set be as above. A collection $\theta=\left\{\left\langle\sum, \theta_{\Sigma}\right\rangle\right.$ $\left.: \sum \in|\operatorname{Sign}|\right\}$ is called an equivalence system of SEN, if

- $\theta_{\Sigma}$ is an equivalence relation on $\operatorname{SEN}(\Sigma)$ for all $\Sigma \in|\operatorname{Sign}|$,

- $\operatorname{SEN}(f)^{2}\left(\theta_{\Sigma_{1}}\right) \subseteq \theta_{\Sigma_{2}}$, for all $\Sigma_{1}, \Sigma_{2} \in|\operatorname{Sign}|, f \in \operatorname{Sign}\left(\Sigma_{1}, \Sigma_{2}\right)$.

If, in addition, $N$ is a category of natural transformations on SEN and $\theta_{\Sigma}$ is an $N$-congruence, for all $\sum \in|\mathbf{S i g n}|$, then $\theta$ is said to be an $N$-congruence system of SEN.

Let now $\mathcal{I}=\left\langle\operatorname{Sign}, \operatorname{SEN},\left\{C_{\sum}\right\}_{\sum \in|\operatorname{Sign}|}\right\rangle$ be a $\pi$-institution. An equivalence system $\theta$ of SEN is called a logical equivalence system of $\mathcal{I}$ if, for all $\Sigma \in|\mathbf{S i g n}|, \phi, \psi \in \operatorname{SEN}(\Sigma)$, 


$$
\langle\phi, \psi\rangle \in \theta_{\Sigma} \quad \text { implies } \quad C_{\Sigma}(\phi)=C_{\Sigma}(\psi) .
$$

An $N$-congruence system of SEN is an $N$-logical congruence system or a logical $N$-congruence system of $\mathcal{I}$ if it is logical as an equivalence system of $\mathcal{I}$.

Sometimes, when the signature $\sum$ is clear from context, the $\sum$-equivalence $\left\langle\Sigma, \theta_{\Sigma}\right\rangle$ or the $\Sigma$-N-congruence $\left\langle\Sigma, \theta_{\Sigma}\right\rangle$ will be denoted simply by $\theta_{\Sigma}$. The same convention will be followed often for $\sum$-theories as well.

Like equivalence relations on sets, equivalence systems on sentence functors form complete lattices under signature-wise inclusions.

Proposition 1. Let Sign be a category and SEN : Sign $\rightarrow$ Set be a functor. The collection $\operatorname{Eqv}(\mathrm{SEN})$ of all equivalence systems of SEN forms a complete lattice $\operatorname{Eqv}(\mathrm{SEN})=\langle\operatorname{Eqv}(\mathrm{SEN}), \leq\rangle$ under signature-wise inclusion $\leq$.

Proof. It is clear that $\nabla=\left\{\left\langle\sum, \nabla_{\Sigma}\right\rangle: \sum \in|\operatorname{Sign}|\right\}$, where $\nabla_{\Sigma}=\operatorname{SEN}(\Sigma)^{2}$, for all $\sum \in|\mathbf{S i g n}|$, is an equivalence system of SEN. So, it is the maximum element of $\operatorname{Eqv}(\mathrm{SEN})$ under signature-wise inclusion.

To prove the statement, it suffices, thus, to show that the collection $\operatorname{Eqv}(\mathrm{SEN})$ is closed under signature-wise intersections. Suppose to this end that $\theta^{i}=\left\{\left\langle\sum, \theta_{\Sigma}^{i}\right\rangle: \Sigma \in|\operatorname{Sign}|\right\}, i \in I$, is a collection of equivalence systems of SEN. Clearly, since $\theta_{\Sigma}^{i}, i \in I$, are all equivalences on $\operatorname{SEN}(\Sigma), \bigcap_{i \in I} \theta_{\Sigma}^{i}$ is also an equivalence relation on $\operatorname{SEN}(\Sigma)$. Therefore, to see that $\bigcap_{i \in I} \theta^{i}=\left\{\left\langle\sum, \bigcap_{i \in I} \theta_{\Sigma}^{i}\right\rangle: \Sigma \in \mid\right.$ Sign $\left.\mid\right\}$ is an equivalence system of SEN, it suffices to show that, for all $\Sigma_{1}, \Sigma_{2} \in|\mathbf{S i g n}|$, $f \in \operatorname{Sign}\left(\Sigma_{1}, \Sigma_{2}\right), \operatorname{SEN}(f)^{2}\left(\bigcap_{i \in I} \theta_{\Sigma_{1}}^{i}\right) \subseteq \bigcap_{i \in I} \theta_{\Sigma_{2}}^{i}$. Indeed, if $\langle\phi, \psi\rangle \in \bigcap_{i \in I} \theta_{\Sigma_{1}}^{i}$, 
then $\langle\phi, \psi\rangle \in \theta_{\Sigma_{1}}^{i}$, for all $i \in I$, whence, since, for all $i \in I, \theta^{i}$ is an equivalence system of $\operatorname{SEN}, \operatorname{SEN}(f)^{2}(\langle\phi, \psi\rangle) \in \theta_{\Sigma_{2}}^{i}$, for all $i \in I$, whence $\operatorname{SEN}(f)^{2}(\langle\phi, \psi\rangle) \in \bigcap_{i \in I} \theta_{\Sigma_{2}}^{i}$, and, hence, $\bigcap_{i \in I} \theta^{i}$ is an equivalence system of SEN.

Furthermore, in analogy with congruences on a given algebra, $\mathrm{N}$-congruence systems on a given sentence functor also form a complete lattice under signature-wise inclusion.

Proposition 2. Let Sign be a category, SEN : Sign $\rightarrow$ Set be a functor and $N$ be a category of natural transformations on SEN. The collection $\mathrm{Con}^{N}(\mathrm{SEN})$ of all $N$-congruence systems on $\mathrm{SEN}$ forms a complete lattice $\operatorname{Con}^{N}(\mathrm{SEN})=\left\langle\operatorname{Con}^{N}(\mathrm{SEN}), \leq\right\rangle$ under signature-wise inclusion $\leq$.

Proof. Since $\nabla=\left\{\left\langle\Sigma, \nabla_{\Sigma}\right\rangle: \Sigma \in|\operatorname{Sign}|\right\}$, where $\nabla_{\Sigma}=\operatorname{SEN}(\Sigma)^{2}$, for all $\sum \in|\mathbf{S i g n}|$, is an $N$-congruence system on SEN, regardless of which category $N$ of natural transformations on SEN is under consideration, it suffices to show that the collection $\mathrm{Con}^{N}(\mathrm{SEN})$ is closed under signature-wise intersections. Suppose, to this end, that $\theta^{i}=\left\{\left\langle\sum, \theta_{\Sigma}^{i}\right\rangle: \Sigma \in|\mathbf{S i g n}|\right\}, i \in I, \quad$ is a collection of $N$-congruence systems of SEN. Clearly, since $\theta_{\Sigma}^{i}, i \in I$, are all equivalences on $\operatorname{SEN}(\Sigma)^{2}, \bigcap_{i \in I} \theta_{\Sigma}^{i}$ is also an equivalence relation on $\operatorname{SEN}(\Sigma)$ and, by Proposition 1, it is preserved by all signature morphisms. Therefore, to see that $\bigcap_{i \in I} \theta^{i}=\left\{\left\langle\sum, \bigcap_{i \in I} \theta_{\Sigma}^{i}\right\rangle: \sum \in|\mathbf{S i g n}|\right\}$ is an $N$-congruence system on SEN, it suffices to show that it is preserved by all $\sigma: \mathrm{SEN}^{k} \rightarrow \mathrm{SEN}$ in $N$. Suppose, to this end, that $\sigma: \mathrm{SEN}^{k} \rightarrow \mathrm{SEN}$ in $N$ and $\vec{\phi}, \vec{\psi} \in \operatorname{SEN}(\Sigma)^{k}$, such that $\vec{\phi}\left(\bigcap_{i \in I} \theta_{\Sigma}^{i}\right)^{k} \vec{\psi}$. Then $\vec{\phi}\left(\theta_{\Sigma}^{i}\right)^{k} \vec{\psi}$, for all $i \in I$. 
Hence, since for all $i \in I, \theta^{i}$ is an $N$-congruence system, $\sigma_{\Sigma}(\vec{\phi}) \theta_{\Sigma}^{i} \sigma_{\Sigma}(\vec{\psi})$, for all $i \in I$. Therefore $\sigma_{\Sigma}(\vec{\phi}) \bigcap_{i \in I} \theta_{\Sigma}^{i} \sigma_{\Sigma}(\vec{\phi})$, whence $\bigcap_{i \in I} \theta^{i}$ is indeed an $N$-congruence system on SEN.

Finally, as in the case of logical congruences on abstract logics, the collection of all logical $N$-congruence systems of a given $\pi$-institution forms a complete lattice under signature-wise inclusion.

Theorem 3. Let $\mathcal{I}=\left\langle\operatorname{Sign}, \mathrm{SEN},\left\{C_{\sum}\right\}_{\sum \in|\operatorname{Sign}|}\right\rangle$ be a $\pi$-institution and $N$ be a category of natural transformations on SEN. The collection $\operatorname{Con}^{N}(\mathcal{I})$, sometimes also denoted by $\operatorname{Con}^{N}(C)$, of all logical $N$-congruence systems of $\mathcal{I}$ forms a complete lattice $\operatorname{Con}^{N}(\mathcal{I})=\left\langle\operatorname{Con}^{N}(\mathcal{I}), \leq\right\rangle$ under signature-wise inclusion $\leq$.

Proof. If $\theta^{i}, i \in I$, with $I \neq \emptyset$, are in $\operatorname{Con}^{N}(\mathcal{I})$, then $\bigcap_{i \in I} \theta^{i} \in \operatorname{Con}^{N}(\mathcal{I})$, since, it is obviously an equivalence system of SEN, it is preserved by every morphism in Sign and by $N$, since every $\theta^{i}, i \in I$, is, and, finally, if $\langle\phi, \psi\rangle \in \bigcap_{i \in I} \theta_{\Sigma}^{i}$, then $\langle\phi, \psi\rangle \in \theta_{\Sigma}^{i}$, for all $i \in I$, whence, since $\theta^{i}$ is a logical $N$-congruence system of $\mathcal{I}, C_{\Sigma}(\phi)=C_{\Sigma}(\psi)$. Therefore, $\bigcap_{i \in I} \theta_{\Sigma}^{i}$ is also a logical $N$-congruence system of $\mathcal{I}$.

It suffices, therefore, to show that $\operatorname{Con}^{N}(\mathcal{I})$ has a greatest element. The signature-wise union of every signature-wise directed subset of $\mathrm{Con}^{N}(\mathcal{I})$ is an upper bound for that subset in $\mathrm{Con}^{N}(\mathrm{SEN})$ and it is in $\operatorname{Con}^{N}(\mathcal{I})$, since every member of the subset is. So, by Zorn's lemma, $\operatorname{Con}^{N}(\mathcal{I})$ has a maximal element. If $\theta \neq \theta^{\prime}$ are two such maximal elements, then, it is not difficult to verify that their join $\eta$ as $N$-congruence systems on SEN is a logical $N$-congruence system of $\mathcal{I}$. 
This, however, contradicts the maximality of $\theta$ and $\theta^{\prime}$, since, clearly, $\theta<\eta$ and $\theta^{\prime}<\eta$. Therefore, the maximal element of $\operatorname{Con}^{N}(\mathcal{I})$ is a largest element.

The largest logical $N$-congruence system is called the Tarski $N$-congruence system of $\mathcal{I}$ and is denoted by $\widetilde{\Omega}^{N}(\mathcal{I})$ or $\widetilde{\Omega}^{N}(C)$.

As a notational convention, when referring to the $U$-Tarski congruence system of a $\pi$-institution $\mathcal{I}$, where $U$ is the category of all natural transformations on SEN, the accompanying superscript $U$ may occasionally be omitted from the notation.

The following theorem is an adaptation of a characterization result of Font and Jansana ([11], Proposition 1.2) of the Tarski congruence of an abstract logic, which is, in turn, a generalization of a characterization result of Blok and Pigozzi [4] of the Leibniz congruence of a logical matrix.

Theorem 4. Let $\mathcal{I}=\left\langle\operatorname{Sign}, \mathrm{SEN},\left\{C_{\sum}\right\}_{\sum \in|\operatorname{Sign}|}\right\rangle$ be a $\pi$-institution, with $N$ a category of natural transformations on $\operatorname{SEN}, \Sigma \in|\operatorname{Sign}|$ and $\phi, \psi \in \operatorname{SEN}(\Sigma)$. Then $\langle\phi, \psi\rangle \in \widetilde{\Omega}_{\Sigma}^{N}(\mathcal{I})$ if and only if, for all $\Sigma \in|\operatorname{Sign}|$, all $f \in \operatorname{Sign}\left(\Sigma, \Sigma^{\prime}\right)$, all natural transformations $\tau: \operatorname{SEN}^{k} \rightarrow \operatorname{SEN}$ in $N$, all $i<k$ and all $\vec{\chi} \in \operatorname{SEN}\left(\sum^{\prime}\right)^{k}$,

$$
\begin{aligned}
C_{\Sigma^{\prime}}\left(\tau_{\Sigma^{\prime}}\left(\chi_{0}, \ldots, \chi_{i-1}, \operatorname{SEN}(f)(\phi), \chi_{i+1}, \ldots, \chi_{k-1}\right)\right. \\
\quad=C_{\Sigma^{\prime}}\left(\tau_{\Sigma^{\prime}}\left(\chi_{0}, \ldots, \chi_{i-1}, \operatorname{SEN}(f)(\psi), \chi_{i+1}, \ldots, \chi_{k-1}\right) .\right.
\end{aligned}
$$

Notational convention. Sometimes, for compactness, Equation (2) will be abbreviated as

$$
C_{\Sigma^{\prime}}\left(\tau_{\Sigma^{\prime}}(\operatorname{SEN}(f)(\phi), \vec{\chi})\right)=C_{\Sigma^{\prime}}\left(\tau_{\Sigma^{\prime}}(\operatorname{SEN}(f)(\psi), \vec{\chi})\right),
$$


with the understanding that $\operatorname{SEN}(f)(\phi), \operatorname{SEN}(f)(\psi)$ may appear in positions other than the first in $\tau_{\Sigma^{\prime}}$, but that they must appear in the same position in both sides of the equation.

Proof. Let $R=\left\{R_{\Sigma}: \sum \in|\mathbf{S i g n}|\right\}$ denote the collection of relations defined by Equation (3). Obviously, $R_{\Sigma}$ is an equivalence relation on $\operatorname{SEN}(\Sigma) . R$ is also an equivalence system of SEN, since, for all $\Sigma_{1}, \Sigma_{2} \in$ $|\operatorname{Sign}|, f \in \operatorname{Sign}\left(\Sigma_{1}, \Sigma_{2}\right)$, and $\langle\phi, \psi\rangle \in R_{\Sigma_{1}}$, we get, for all $\Sigma^{\prime} \in|\operatorname{Sign}|$, $g \in \operatorname{Sign}\left(\Sigma_{2}, \Sigma^{\prime}\right)$,

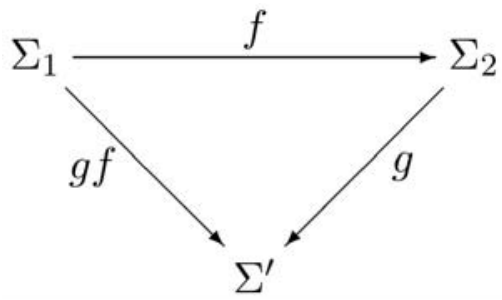

$$
\begin{aligned}
& \tau: \operatorname{SEN}^{k} \rightarrow \operatorname{SEN} \text { in } N \text { and } \vec{\chi} \in \operatorname{SEN}\left(\Sigma^{\prime}\right)^{k}, \\
& C_{\Sigma}\left(\tau_{\Sigma^{\prime}}(\operatorname{SEN}(g)(\operatorname{SEN}(f)(\phi)), \vec{\chi})\right) \quad=\quad C_{\Sigma^{\prime}}\left(\tau_{\Sigma^{\prime}}(\operatorname{SEN}(g f)(\phi), \vec{\chi})\right) \\
& =\quad C_{\Sigma^{\prime}}\left(\tau \Sigma_{\Sigma^{\prime}}(\operatorname{SEN}(g f)(\psi), \vec{\chi})\right) \\
& =\quad C_{\Sigma^{\prime}}\left(\tau_{\Sigma^{\prime}}(\operatorname{SEN}(g)(\operatorname{SEN}(f)(\psi)), \vec{\chi})\right) \text {, }
\end{aligned}
$$

where, passing from the first to the second lines above, we have used the fact that $\langle\phi, \psi\rangle \in R_{\Sigma_{1}}$ and $g f \in \operatorname{Sign}\left(\Sigma_{1}, \Sigma^{\prime}\right)$. Therefore, $\operatorname{SEN}(f)^{2}(\langle\phi, \psi\rangle)$ $\in R_{\Sigma_{2}}$. Furthermore, $R$ is an $N$-congruence system on SEN. To see this, let $\sum \in|\operatorname{Sign}|, \vec{\phi}, \vec{\psi} \in \operatorname{SEN}\left(\sum\right)^{n}$, such that $\vec{\phi} R_{\Sigma}^{n} \vec{\psi}$, and $\sigma: \operatorname{SEN}^{n} \rightarrow \operatorname{SEN}$ be in $N$. Then, for all $\Sigma^{\prime} \in|\operatorname{Sign}|, f \in \operatorname{Sign}\left(\sum, \Sigma^{\prime}\right), \tau: \operatorname{SEN}^{k} \rightarrow \operatorname{SEN}$ in $N$ and $\vec{\chi} \in \operatorname{SEN}\left(\sum^{\prime}\right)^{k}$, 


$$
\begin{aligned}
C_{\Sigma^{\prime}}\left(\tau_{\Sigma^{\prime}}\left(\operatorname{SEN}(f)\left(\sigma_{\Sigma}(\vec{\phi})\right), \vec{\chi}\right)\right) & =C_{\Sigma^{\prime}}\left(\tau_{\Sigma^{\prime}}\left(\sigma_{\Sigma^{\prime}}\left(\operatorname{SEN}(f)^{n}(\vec{\phi})\right), \vec{\chi}\right)\right) \\
& =C_{\Sigma^{\prime}}\left(\tau_{\Sigma^{\prime}}\left(\sigma_{\Sigma^{\prime}}\left(\operatorname{SEN}(f)^{n}(\vec{\psi})\right), \vec{\chi}\right)\right) \\
& =C_{\Sigma^{\prime}}\left(\tau_{\Sigma^{\prime}}\left(\operatorname{SEN}(f)\left(\sigma_{\Sigma}(\vec{\psi})\right), \vec{\chi}\right)\right),
\end{aligned}
$$

where, passing from the first to the second line, we have used the fact that $\tau(\sigma, \ldots): \operatorname{SEN}^{n+k-1} \rightarrow \operatorname{SEN}$ is in $N$ and $\vec{\phi} R_{\Sigma}^{n} \vec{\psi}$. Thus, $\left\langle\sigma_{\Sigma}(\vec{\phi}), \sigma_{\Sigma}\right.$ $(\vec{\psi})\rangle \in R_{\Sigma}$ and $R$ is an $N$-congruence system on SEN. Finally, it is straightforward to see, taking the identity morphism $i_{\Sigma}: \sum \rightarrow \sum$ and the identity natural transformation $\iota: \operatorname{SEN} \rightarrow$ SEN (which is in $N$ ) for $f$ and $\tau$, respectively, in Equation (3), that $R$ is a logical $N$-congruence system of $\mathcal{I}$. Therefore, by the definition of $\widetilde{\Omega} \Sigma_{\Sigma}^{N}(\mathcal{I})$, we get that $R_{\Sigma} \subseteq \widetilde{\Omega}_{\Sigma}^{N}(\mathcal{I})$, for all $\Sigma \in|\operatorname{Sign}|$.

Conversely, if $\langle\phi, \psi\rangle \in \widetilde{\Omega}_{\Sigma}^{N}(\mathcal{I})$, then, since $\widetilde{\Omega}^{N}(\mathcal{I})$ is an equivalence system of SEN, we get, for every $\Sigma^{\prime} \in|\operatorname{Sign}|, f \in \operatorname{Sign}\left(\Sigma, \Sigma^{\prime}\right)$,

$$
\langle\operatorname{SEN}(f)(\phi), \operatorname{SEN}(f)(\psi)\rangle \in \widetilde{\Omega}_{\Sigma^{\prime}}^{N}(\mathcal{I}) .
$$

Now, since $\widetilde{\Omega}_{\Sigma}^{N}(\mathcal{I})$ is an equivalence relation on $\operatorname{SEN}\left(\Sigma^{\prime}\right)$, we get, for every $\vec{\chi} \in \operatorname{SEN}\left(\sum^{\prime}\right)^{k}, \vec{\chi} \widetilde{\Omega}{\widetilde{\Sigma^{\prime}}}^{N}(\mathcal{I})^{k} \vec{\chi}$, whence, since $\widetilde{\Omega}^{N}(\mathcal{I})$ is an $N$-congruence system, we get, for every $\tau: \mathrm{SEN}^{k} \rightarrow \mathrm{SEN}$ in $N$,

$$
\left\langle\tau_{\Sigma^{\prime}}(\operatorname{SEN}(f)(\phi), \vec{\chi}), \tau_{\Sigma^{\prime}}(\operatorname{SEN}(f)(\psi), \vec{\chi})\right\rangle \in \widetilde{\Omega}_{\Sigma^{\prime}}^{N}(\mathcal{I}) .
$$

Therefore, since $\widetilde{\Omega}^{N}(\mathcal{I})$ is a logical $N$-congruence system of $\mathcal{I}$, we have

$$
C_{\Sigma^{\prime}}\left(\tau_{\Sigma^{\prime}}(\operatorname{SEN}(f)(\phi), \vec{\chi})\right)=C_{\Sigma^{\prime}}\left(\tau_{\Sigma^{\prime}}(\operatorname{SEN}(f)(\psi), \vec{\chi})\right) .
$$

Hence $\langle\phi, \varphi\rangle \in R_{\Sigma}$ and $\widetilde{\Omega}_{\Sigma}^{N}(\mathcal{I}) \subseteq R_{\Sigma}$ for every $\sum \in|\operatorname{Sign}|$. This concludes the proof that $R=\widetilde{\Omega}^{N}(\mathcal{I})$. 
In the sequel, it is shown how the results of Blok and Pigozzi [4] and Font and Jansana [11] become special cases of the results presented above. To this end, given a language or similarity type $\mathcal{L}$, an $\mathcal{L}$-algebra $\mathbf{A}=\left\langle A, \mathcal{L}^{\mathbf{A}}\right\rangle$ and an abstract logic $\mathbb{L}=\langle\mathbf{A}, C\rangle$, denote by $\mathcal{I}^{\mathbb{L}}$ the $\pi$-institution that has as its signature category the trivial category with the object $\mathbf{A}$, as its sentence functor the functor sending the algebra $\mathbf{A}$ to its underlying universe $A$ and as its closure system the closure system defined by $C_{\mathbf{A}}=C: \mathcal{P}(A) \rightarrow \mathcal{P}(A)$. It is not difficult to check that this is indeed a $\pi$-institution, which will be referred to as the $\pi$-institution associated with the abstract logic $\mathbb{L}$. Note that the clone $N$ of algebraic operations generated by $\mathcal{L}^{\mathbf{A}}$ forms a category of natural transformations on SEN in the sense of the present paper. The notation just introduced will be kept for the remainder of this section as the results above are related to the results of Blok and Pigozzi and of Font and Jansana.

The following corollary of Theorem 3 is the result of Blok and Pigozzi for universal algebraic congruences compatible with given filters on their algebras.

Corollary 5 (Blok-Pigozzi). Let $\mathbf{A}=\left\langle A, \mathcal{L}^{\mathbf{A}}\right\rangle$ be an $\mathcal{L}$-algebra and $F \subseteq A$. The collection of all $\mathcal{L}$-congruences that are compatible with $F$ forms a complete lattice under inclusion.

Proof. Consider the $\pi$-institution $\mathcal{I}^{\mathbb{L}}$ associated with the abstract logic $\mathbb{L}=\left\langle\left\langle A, \mathcal{L}^{\mathbf{A}}\right\rangle, C\right\rangle$, where $C$ has $F$ and $A$ as its only two theories. Let $N$ be the category of natural transformations on SEN corresponding to the clone of $\mathcal{L}^{\mathbf{A}}$. Then the result follows from Theorem 3.

The largest $\mathcal{L}$-congruence that is compatible with a given subset $F$ is called the Leibniz congruence of $\langle\mathbf{A}, F\rangle$ and is denoted by $\Omega_{\mathbf{A}}(F)$. The name has been introduced by the inventors of the congruence, Blok and Pigozzi, and the reader may find an excellent account of some of its properties and of its rôle in abstract algebraic logic in their seminal "Memoirs monograph" [4]. 
Theorem 3 has also as a corollary the following result of Font and Jansana ([11], Formula (1.2)).

Corollary 6 (Font-Jansana). Let $\mathbb{L}=\langle\mathbf{A}, C\rangle$ be an abstract logic. The collection of all logical congruences of $\mathbb{L}$ forms a complete lattice under inclusion.

Proof. Very similar to the proof of Corollary 5.

The largest logical congruence is called the Tarski congruence of $\mathbb{L}$ and is denoted by $\widetilde{\Omega}_{\mathrm{A}}(C)$. The name has been introduced by the inventors of the congruence, Josep Maria Font and Ramon Jansana, and the reader may also find a fascinating treatment of its properties and rôle in abstract algebraic logic in their excellent treatise [11].

Theorem 4 has the following consequence. It is based on a result of Blok and Pigozzi characterizing the Leibniz congruence associated with a theory of a logic. Font and Jansana (see [11], Formula (1.3)) adapted the result and used it to characterize the Tarski congruence associated with an abstract logic. In the current version, the Tarski congruence system of the $\pi$-institution $\mathcal{I}^{\mathbb{L}}$ associated with an abstract logic $\mathbb{L}=\langle\mathbf{A}, C\rangle$, which can be identified with the Tarski congruence of the abstract logic, is characterized.

Corollary 7 (Font-Jansana). Let $\mathcal{I}^{\mathbb{L}}=\left\langle\operatorname{Sign}, \mathrm{SEN},\left\{C_{\sum}\right\}_{\sum \in|\operatorname{Sign}|}\right\rangle$ be the $\pi$-institution associated with the abstract logic $\mathbb{L}=\left\langle\left\langle A, \mathcal{L}^{\mathbf{A}}\right\rangle, C\right\rangle$, and $N$ be the category of natural transformations on SEN corresponding to the clone of $\mathcal{L}^{\mathbf{A}}$ operations. Then, for all $a, b \in A,\langle a, b\rangle \in \widetilde{\Omega}_{\mathbf{A}}^{N}(C)$ if and only if, for every $\mathcal{L}$-formula $\phi(p, \vec{q})$ and every $\vec{c} \in A^{k}$, where $k$ is the length of $\vec{q}$,

$$
C_{\mathbf{A}}\left(\phi^{\mathbf{A}}(a, \vec{c})\right)=C_{\mathbf{A}}\left(\phi^{\mathbf{A}}(b, \vec{c})\right) .
$$


It is appropriate to pause here to pay tribute to the aforementioned works of Blok and Pigozzi [4] and of Font and Jansana [11], without which our work would not have existed. Also of equal importance has been the work of Janusz Czelakowski [7], that paved the way for the work of Blok and Pigozzi. More recently, Czelakowski has written an excellent overview of abstract algebraic logic [8]. All three works have been extremely important for abstract algebraic logic in general and, in particular, in placing an appropriate emphasis on the key rôle of congruences with logical compatibility properties.

\section{Logical and Bilogical Morphisms}

Let $\mathcal{I}=\left\langle\right.$ Sign, SEN, $\left.\left\{C_{\sum}\right\}_{\sum \in|\operatorname{Sign}|}\right\rangle, \mathcal{I}=\left\langle\right.$ Sign' $\left.^{\prime}, \operatorname{SEN}^{\prime},\left\{C_{\sum}^{\prime}\right\}_{\sum \in\left|\mathbf{S i g n}^{\prime}\right|}\right\rangle$ be two $\pi$-institutions. A translation $\langle F, a\rangle: \mathcal{I} \rightarrow \mathcal{I}^{\prime}$ from $\mathcal{I}$ to $\mathcal{I}^{\prime}$ [21] is a pair consisting of a functor $F:$ Sign $\rightarrow$ Sign' and a natural transformation $\alpha: \mathrm{SEN} \rightarrow \mathcal{P S E N} F$. A translation is said to be a singleton translation, denoted $\langle F, a\rangle: \mathcal{I} \rightarrow^{s} \mathcal{I}^{\prime}$, if, for all $\sum \in|\operatorname{Sign}|$, $\phi \in \operatorname{SEN}(\Sigma),\left|\alpha_{\Sigma}(\phi)\right|=1$. In that case, the set $\alpha_{\Sigma}(\phi)$ will be identified with the only element that it contains and $\alpha$ will be treated as a natural transformation $\alpha:$ SEN $\rightarrow \mathrm{SEN}^{\prime} F$.

A singleton translation $\langle F, a\rangle: \mathcal{I} \rightarrow^{s} \mathcal{I}^{\prime}$ is said to be surjective, if

(1) $F:$ Sign $\rightarrow$ Sign' $^{\prime}$ is surjective and

(2) $\alpha_{\Sigma}: \operatorname{SEN}(\Sigma) \rightarrow \operatorname{SEN}^{\prime}\left(F\left(\sum\right)\right)$ is surjective, for all $\sum \in|\operatorname{Sign}|$.

If only condition (2) above holds, $\langle F, a\rangle$ will be said to be $\alpha$-surjective.

A translation $\langle F, a\rangle: \mathcal{I} \rightarrow \mathcal{I}^{\prime}$ is a semi-interpretation from $\mathcal{I}$ to $\mathcal{I}^{\prime}$, written $\langle F, a\rangle: \mathcal{I}\rangle-\mathcal{I}^{\prime}$, if, for every $\Sigma \in|\operatorname{Sign}|, \Phi \cup\{\phi\} \subseteq \operatorname{SEN}(\Sigma)$,

$$
\phi \in C_{\Sigma}(\Phi) \text { implies } \alpha_{\Sigma}(\Phi) \subseteq C_{F(\Sigma)}^{\prime}\left(\alpha_{\Sigma}(\Phi)\right) .
$$


It is an interpretation from $\mathcal{I}$ to $\mathcal{I}^{\prime}$, denoted $\langle F, a\rangle: \mathcal{I} \vdash \mathcal{I}^{\prime}$, if, for all $\sum \in|\operatorname{Sign}|, \Phi \cup\{\phi\} \subseteq \operatorname{SEN}(\Sigma)$,

$$
\phi \in C_{\Sigma}(\Phi) \text { iff } \quad \alpha_{\Sigma}(\phi) \subseteq C_{F(\Sigma)}^{\prime}\left(\alpha_{\Sigma}(\Phi)\right) .
$$

Let SEN : Sign $\rightarrow$ Set be a sentence functor and $N$ be a category of natural transformations on SEN. A functor SEN' : Sign' $\rightarrow$ Set, with a category $N^{\prime}$ of natural transformations on $\mathrm{SEN}^{\prime}$ is called an $N$-(sentence) functor if there exists a surjective functor $: N \rightarrow N^{\prime}$ that preserves all projection natural transformations and, as a result, also the arities of all natural transformations. In this case, the image of $\sigma: \mathrm{SEN}^{k} \rightarrow \mathrm{SEN}$ in $N$ under' will be denoted by $\sigma^{\prime}: \mathrm{SEN}^{\prime k} \rightarrow \mathrm{SEN}^{\prime}$. Similar conventions will be adopted throughout without explicit mention.

Given $N$-functors $\mathrm{SEN}^{\prime}:$ Sign' $^{\prime} \rightarrow$ Set and SEN" : Sign" $\rightarrow$ Set, with categories $N^{\prime}, N^{\prime \prime}$ of natural transformations on $\mathrm{SEN}^{\prime}, \mathrm{SEN}^{\prime \prime}$, respectively, an $\left(N^{\prime}, N^{\prime \prime}\right)$-morphism $\langle F, \alpha\rangle: \mathrm{SEN}^{\prime} \rightarrow \mathrm{SEN}^{\prime \prime}$ consists of

(1) a functor $F:$ Sign' $^{\rightarrow}$ Sign" and

(2) a natural transformation $\alpha: \mathrm{SEN}^{\prime} \rightarrow \mathrm{SEN}^{\prime \prime} F$,

satisfying, for all $\tau: \operatorname{SEN}^{k} \rightarrow \operatorname{SEN}$ in $N$, all $\sum \in\left|\mathbf{S i g n}^{\prime}\right|$ and all $\vec{\phi} \in \operatorname{SEN}^{\prime}(\Sigma)^{k}$,

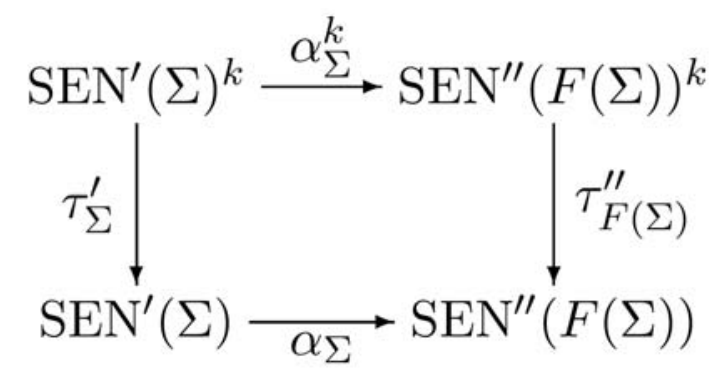

$$
\alpha_{\Sigma}\left(\tau_{\Sigma}^{\prime}(\vec{\phi})\right)=\tau_{F(\Sigma)}^{\prime \prime}\left(\alpha_{\Sigma}^{k}(\vec{\phi})\right)
$$


We assume in the sequel that all our sentence functors are $\left(N^{\prime}, N^{\prime \prime}\right)$-functors, with reference to some fixed category $N^{b}$ of natural transformations on a fixed base sentence functor $\operatorname{SEN}^{b}: \mathbf{S i g n}^{b} \rightarrow$ Set.

Given $\pi$-institutions $\mathcal{I}=\left\langle\mathbf{S i g n}, \mathrm{SEN},\left\{C_{\sum}\right\}_{\sum \in|\operatorname{Sign}|}\right\rangle$ and $\mathcal{I}^{\prime}=\left\langle\mathbf{S i g n}^{\prime}\right.$, $\left.\mathrm{SEN}^{\prime},\left\{C_{\sum}^{\prime}\right\}_{\sum \in\left|\mathbf{S i g n}^{\prime}\right|}\right\rangle$ and categories of natural transformations $N, N^{\prime}$, respectively, on $\mathrm{SEN}, \mathrm{SEN}^{\prime}$, a singleton translation (semi-interpretation or interpretation) $\langle F, \alpha\rangle$ from $\mathcal{I}$ to $\mathcal{I}^{\prime}$ is said to be an $\left(N, N^{\prime}\right)$-translation (semi-interpretation or interpretation) if, in addition, $\langle F, \alpha\rangle: \mathrm{SEN} \rightarrow \mathrm{SEN}^{\prime}$ is an $\left(N, N^{\prime}\right)$-morphism.

An $\left(N, N^{\prime}\right)$-logical morphism $\langle F, \alpha\rangle: \mathcal{I} \rightarrow \mathcal{I}^{\prime}$ from $\mathcal{I}$ to $\mathcal{I}^{\prime}$ is a (singleton) $\left(N, N^{\prime}\right)$-semi-interpretation from $\mathcal{I}$ to $\mathcal{I}^{\prime}$, denoted accordingly by $\langle F, \alpha\rangle: \mathcal{I}\rangle{ }^{s} \mathcal{I}^{\prime}$. A logical morphism is strong if it is an interpretation, denoted $\langle F, \alpha\rangle: \mathcal{I} \vdash^{s} \mathcal{I}^{\prime}$. Finally, it is called an $\left(N, N^{\prime}\right)$-bilogical morphism if it is a surjective strong $\left(N, N^{\prime}\right)$-logical morphism and an isomorphism if $F:$ Sign $\rightarrow$ Sign' $^{\prime}$ and $\alpha_{\Sigma}: \operatorname{SEN}\left(\sum\right) \rightarrow \operatorname{SEN}^{\prime}\left(F\left(\sum\right)\right)$, for all $\sum \in|\operatorname{Sign}|$, are isomorphisms.

The following proposition gives a preservation property of the $N$-Tarski congruence system under the action of logical morphisms.

Proposition 8. Suppose that $\mathcal{I}=\left\langle\operatorname{Sign}, \mathrm{SEN},\left\{C_{\sum}\right\}_{\sum \in|\operatorname{Sign}|}\right\rangle$ and $\mathcal{I}^{\prime}=\left\langle\right.$ Sign' $\left.^{\prime}, \mathrm{SEN}^{\prime},\left\{C_{\sum}^{\prime}\right\}_{\sum \in\left|\mathbf{S i g n}^{\prime}\right|}\right\rangle$ are two $\pi$-institutions, $N, N^{\prime}$ are categories of natural transformations on $\mathrm{SEN}, \mathrm{SEN}^{\prime}$, respectively, and $\langle F, \alpha\rangle: \mathcal{I}\rangle-{ }^{s} \mathcal{I}^{\prime}$ is an $\left(N, N^{\prime}\right)$-logical morphism, such that $\langle F, \alpha\rangle$ is surjective. Then, for every $\sum \in|\mathbf{S i g n}|$,

$$
\alpha_{\Sigma}\left(\widetilde{\Omega}_{\Sigma}^{N}(C)\right) \subseteq \widetilde{\Omega}_{F(\Sigma)}^{N^{\prime}}\left(C^{\prime}\right)
$$


Proof. Let $\Sigma \in|\operatorname{Sign}|, \phi, \psi \in \operatorname{SEN}(\Sigma)$. Suppose that $\langle\phi, \psi\rangle \in \widetilde{\Omega}_{\Sigma}^{N}(C)$. Then, by Theorem 4 , for all $\Sigma^{\prime} \in|\operatorname{Sign}|, f \in \operatorname{Sign}\left(\Sigma, \Sigma^{\prime}\right), \tau: \operatorname{SEN}^{k} \rightarrow \operatorname{SEN}$ in $N$ and $\vec{\chi} \in \operatorname{SEN}\left(\sum^{\prime}\right)^{k}$, we have

$$
C_{\Sigma^{\prime}}\left(\tau_{\Sigma^{\prime}}(\operatorname{SEN}(f)(\phi), \vec{\chi})\right)=C_{\Sigma^{\prime}}\left(\tau_{\Sigma^{\prime}}(\operatorname{SEN}(f)(\psi), \vec{\chi})\right),
$$

whence $\alpha_{\Sigma^{\prime}}\left(C_{\Sigma^{\prime}}\left(\tau_{\Sigma^{\prime}}(\operatorname{SEN}(f)(\phi), \vec{\chi})\right)\right)=\alpha_{\Sigma^{\prime}}\left(C_{\Sigma^{\prime}}\left(\tau_{\Sigma^{\prime}}(\operatorname{SEN}(f)(\psi), \vec{\chi})\right)\right)$. Thus, by Lemma 6.4 of [21], which is expressed for interpretations but is also valid for semi-interpretations,

$$
C_{F\left(\Sigma^{\prime}\right)}^{\prime}\left(\alpha_{\Sigma^{\prime}}\left(\tau_{\Sigma^{\prime}}(\operatorname{SEN}(f)(\phi), \vec{\chi})\right)\right)=C_{F\left(\Sigma^{\prime}\right)}^{\prime}\left(\alpha_{\Sigma^{\prime}}\left(\tau_{\Sigma^{\prime}}(\operatorname{SEN}(f)(\psi), \vec{\chi})\right)\right) .
$$

But then, since $\langle F, \alpha\rangle$ is an $\left(N, N^{\prime}\right)$-morphism,

$$
C_{F\left(\Sigma^{\prime}\right)}^{\prime}\left(\tau_{F\left(\Sigma^{\prime}\right)}^{\prime}\left(\alpha_{\Sigma^{\prime}}^{k}(\operatorname{SEN}(f)(\phi), \vec{\chi})\right)\right)=C_{F\left(\Sigma^{\prime}\right)}^{\prime}\left(\tau_{F\left(\Sigma^{\prime}\right)}^{\prime}\left(\alpha_{\Sigma^{\prime}}^{k}(\operatorname{SEN}(f)(\psi), \vec{\chi})\right)\right)
$$

i.e.,

$C_{F\left(\Sigma^{\prime}\right)}^{\prime}\left(\tau_{F\left(\Sigma^{\prime}\right)}^{\prime}\left(\alpha_{\Sigma^{\prime}}(\operatorname{SEN}(f)(\phi)), \alpha_{\Sigma^{\prime}}^{k}(\vec{\chi})\right)\right)$

$$
=C_{F\left(\Sigma^{\prime}\right)}^{\prime}\left(\tau_{F\left(\Sigma^{\prime}\right)}^{\prime}\left(\alpha_{\Sigma^{\prime}}(\operatorname{SEN}(f)(\psi)), \alpha_{\Sigma^{\prime}}^{k}(\vec{\chi})\right)\right) .
$$

Hence,

$$
\begin{aligned}
C_{F\left(\Sigma^{\prime}\right)}^{\prime}\left(\tau_{F\left(\Sigma^{\prime}\right)}^{\prime}\left(\operatorname{SEN}^{\prime}(F(f))\left(\alpha_{\Sigma}(\phi)\right), \alpha_{\Sigma^{\prime}}^{k}(\vec{\chi})\right)\right) & \\
& =C_{F\left(\Sigma^{\prime}\right)}^{\prime}\left(\tau_{F\left(\Sigma^{\prime}\right)}^{\prime}\left(\operatorname{SEN}^{\prime}(F(f))\left(\alpha_{\Sigma}(\psi)\right), \alpha_{\Sigma^{\prime}}^{k}(\vec{\chi})\right)\right) .
\end{aligned}
$$

Since $\langle F, \alpha\rangle$ is a surjective $\left(N, N^{\prime}\right)$-morphism, this yields that

$$
C_{\Sigma^{\prime}}^{\prime}\left(\sigma_{\Sigma^{\prime}}\left(\operatorname{SEN}^{\prime}\left(f^{\prime}\right)\left(\alpha_{\Sigma}(\phi)\right), \vec{\chi}^{\prime}\right)\right)=C_{\Sigma^{\prime}}^{\prime}\left(\sigma_{\Sigma^{\prime}}\left(\operatorname{SEN}^{\prime}\left(f^{\prime}\right)\left(\alpha_{\Sigma}(\psi)\right), \vec{\chi}^{\prime}\right)\right)
$$

for all $\Sigma^{\prime} \in\left|\mathbf{S i g n}^{\prime}\right|, f^{\prime} \in \operatorname{Sign}^{\prime}\left(F(\Sigma), \Sigma^{\prime}\right), \sigma^{\prime}: \mathrm{SEN}^{\prime k} \rightarrow \mathrm{SEN}^{\prime}$ in $N^{\prime}$ and $\vec{\chi}^{\prime} \in \operatorname{SEN}^{\prime}\left(\Sigma^{\prime}\right)^{k}$. Therefore, again by Theorem $4,\langle\phi, \psi\rangle \in \widetilde{\Omega}_{F(\Sigma)}^{N^{\prime}}\left(C^{\prime}\right)$. 
The preservation property of Proposition 8 translates to the following monotonicity property of the Tarski congruence systems of two closure operators acting on the same sentence functor. Corollary 9 generalizes Proposition 1.3 of [11].

Corollary 9. Suppose that $\mathcal{I}=\left\langle\right.$ Sign, SEN,$\left.\left\{C_{\sum}\right\}_{\Sigma \in \mid \text { Sign } \mid}\right\rangle, \mathcal{I}^{\prime}=\langle$ Sign, $\left.\mathrm{SEN},\left\{C_{\sum}^{\prime}\right\}_{\sum \in\left|\mathbf{S i g n}^{\prime}\right|}\right\rangle$ are two $\pi$-institutions with the same signature and sentence functors, such that $C \leq C^{\prime}$, i.e., for all $\sum \in|\mathbf{S i g n}|$, $\Phi \subseteq \operatorname{SEN}(\Sigma), C_{\Sigma}(\Phi) \subseteq C_{\Sigma}^{\prime}(\Phi)$. Let also $N$ be a category of natural transformations on SEN. Then, for every $\sum \in|\operatorname{Sign}|, \widetilde{\Omega}_{\Sigma}^{N}(C) \subseteq \widetilde{\Omega}_{\Sigma}^{N}\left(C^{\prime}\right)$, i.e., $\widetilde{\Omega}^{N}(C) \leq \widetilde{\Omega}^{N}\left(C^{\prime}\right)$.

The following lemma provides a characterization of those singleton translations that are semi-interpretations in terms of their behavior on theories. Lemma 13, following Corollary 11, gives a similar characterization for interpretations. By adding the hypothesis that the translations respect natural transformations, one obtains as corollaries similar characterizations for logical morphisms and strong logical morphisms.

Lemma 10. Suppose that $\mathcal{I}=\left\langle\mathbf{S i g n}, \mathrm{SEN},\left\{C_{\sum}\right\}_{\sum \in|\operatorname{Sign}|}\right\rangle$ and $\mathcal{I}^{\prime}=\left\langle\mathbf{S i g n}^{\prime}, \mathrm{SEN}^{\prime},\left\{C_{\sum}^{\prime}\right\}_{\Sigma \in\left|\mathbf{S i g n}^{\prime}\right|}\right\rangle$ are two $\pi$-institutions and $\langle F, \alpha\rangle: \mathcal{I} \rightarrow^{s} \mathcal{I}^{\prime}$ is a singleton translation. $\langle F, \alpha\rangle$ is a singleton semi-interpretation $\langle F, \alpha\rangle: \mathcal{I}\rangle-^{s} \mathcal{I}^{\prime}$ if and only if, for all $\Sigma \in|\mathbf{S i g n}|$ and every $F(\Sigma)$-theory $T^{\prime}$ of $\mathcal{I}^{\prime}, \alpha_{\Sigma}^{-1}\left(T^{\prime}\right)$ is a $\sum$-theory of $\mathcal{I}$.

Proof. First, assume that, for all $\Sigma \in \mid$ Sign $\mid$ and all $F(\Sigma)$-theories $T^{\prime}$ of $\mathcal{I}^{\prime}, \alpha_{\Sigma}^{-1}\left(T^{\prime}\right)$ is a $\sum$-theory of $\mathcal{I}$ and $\phi \in C_{\Sigma}(\Phi)$. Since $\Phi \subseteq \alpha_{\Sigma}^{-1}$ $\left(C_{F(\Sigma)}\left(\alpha_{\Sigma}(\Phi)\right)\right)$, we have $C_{\Sigma}(\Phi) \subseteq \alpha_{\Sigma}^{-1}\left(C_{F(\Sigma)}\left(\alpha_{\Sigma}(\Phi)\right)\right)$ and hence

$$
\alpha_{\Sigma}(\phi) \in \alpha_{\Sigma}\left(C_{\Sigma}(\Phi)\right) \subseteq C_{F(\Sigma)}\left(\alpha_{\Sigma}(\Phi)\right)
$$


Assume, conversely, that $T^{\prime}$ is an $F\left(\sum\right)$-theory of $\mathcal{I}^{\prime}$ and, also, that $\phi \in C_{\Sigma}\left(\alpha_{\Sigma}^{-1}\left(T^{\prime}\right)\right)$. Then $\alpha_{\Sigma}(\phi) \in \alpha_{\Sigma}\left(C_{\Sigma}\left(\alpha_{\Sigma}^{-1}\left(T^{\prime}\right)\right)\right)$, whence

$$
\alpha_{\Sigma}(\phi) \in C_{F(\Sigma)}\left(\alpha_{\Sigma}\left(\alpha_{\Sigma}^{-1}\left(T^{\prime}\right)\right)\right) \subseteq C_{F(\Sigma)}\left(T^{\prime}\right)=T^{\prime},
$$

and, therefore, $\phi \in \alpha_{\Sigma}^{-1}\left(T^{\prime}\right)$. This proves that $\alpha_{\Sigma}^{-1}\left(T^{\prime}\right)$ is a $\sum$-theory.

Corollary 11. Let $\mathcal{I}=\left\langle\right.$ Sign, $\left.\operatorname{SEN},\left\{C_{\sum}\right\}_{\sum \in|\operatorname{Sign}|}\right\rangle$ and $\mathcal{I}^{\prime}=\left\langle\mathbf{S i g n}^{\prime}\right.$, $\left.\mathrm{SEN}^{\prime},\left\{C_{\sum}^{\prime}\right\}_{\Sigma \in\left|\operatorname{Sign}^{\prime}\right|}\right\rangle$ be $\pi$-institutions, $N, N^{\prime}$ be categories of natural transformations on $\mathrm{SEN}$ and $\mathrm{SEN}^{\prime}$, respectively, and $\langle F, \alpha\rangle: \mathcal{I} \rightarrow^{s} \mathcal{I}^{\prime}$ be an $\left(N, N^{\prime}\right)$-translation. $\langle F, \alpha\rangle$ is an $\left(N, N^{\prime}\right)$-logical morphism if and only if, for all $\Sigma \in|\operatorname{Sign}|$ and every $F(\Sigma)$-theory $T^{\prime}$ of $\mathcal{I}^{\prime}, \alpha_{\Sigma}^{-1}\left(T^{\prime}\right)$ is a $\sum$-theory of $\mathcal{I}$.

Next, interpretations from $\mathcal{I}$ to $\mathcal{I}^{\prime}$ are characterized via a similar condition on their action on theories.

Lemma 12. Suppose that $\mathcal{I}=\left\langle\right.$ Sign, SEN, $\left.\left\{C_{\sum}\right\}_{\sum \in|\operatorname{Sign}|}\right\rangle$ and $\mathcal{I}^{\prime}=\left\langle\mathbf{S i g n}^{\prime}, \mathrm{SEN}^{\prime},\left\{C_{\Sigma}^{\prime}\right\}_{\sum \in\left|\mathbf{S i g n}^{\prime}\right|}\right\rangle$ are two $\pi$-institutions and $\langle F, \alpha\rangle: \mathcal{I} \rightarrow^{s} \mathcal{I}^{\prime}$ is a singleton translation. $\langle F, \alpha\rangle$ is a singleton interpretation $\langle F, \alpha\rangle: \mathcal{I} \vdash^{s} \mathcal{I}^{\prime}$ if and only if, for all $\Sigma \in|\mathbf{S i g n}|$,

$$
\left\{\alpha_{\Sigma}^{-1}\left(T^{\prime}\right): T^{\prime} \text { is an } F(\Sigma) \text {-theory }\right\}
$$

is the set of all $\sum$-theories of $\mathcal{I}$, i.e., iff $\operatorname{Th}_{\Sigma}(\mathcal{I})=\alpha_{\Sigma}^{-1}\left(\operatorname{Th}_{F(\Sigma)}\left(\mathcal{I}^{\prime}\right)\right)$.

Proof. Suppose, first, that $\langle F, \alpha\rangle: \mathcal{I} \vdash^{s} \mathcal{I}^{\prime}$. Then, we do have $\langle F, \alpha\rangle: \mathcal{I}\rangle-^{s} \mathcal{I}^{\prime}$, whence, by Lemma 10 , we get $\alpha_{\Sigma}^{-1}\left(\operatorname{Th}_{F(\Sigma)}\left(\mathcal{I}^{\prime}\right)\right) \subseteq \operatorname{Th}_{\Sigma}(\mathcal{I})$. Therefore, it suffices to show that $\operatorname{Th}_{\Sigma}(\mathcal{I}) \subseteq \alpha_{\Sigma}^{-1}\left(\operatorname{Th}_{F(\Sigma)}\left(\mathcal{I}^{\prime}\right)\right)$. To see that this is true, let $T$ be a $\sum$-theory of $\mathcal{I}$. It will be shown that $T=\alpha_{\Sigma}^{-1}\left(C_{F(\Sigma)}^{\prime}\left(\alpha_{\Sigma}(T)\right)\right)$. We do indeed have, for all $\phi \in \operatorname{SEN}(\Sigma)$, 


$$
\begin{array}{lll}
\phi \in \alpha_{\Sigma}^{-1}\left(C_{F(\Sigma)}^{\prime}\left(\alpha_{\Sigma}(T)\right)\right) \quad & \text { iff } & \alpha_{\Sigma}(\phi) \in C_{F(\Sigma)}^{\prime}\left(\alpha_{\Sigma}(T)\right) \\
& \text { iff } \quad \phi \in C_{\Sigma}(T) \\
& \text { iff } \quad \phi \in T .
\end{array}
$$

Suppose, conversely, that $\alpha_{\Sigma}^{-1}\left(\operatorname{Th}_{F(\Sigma)}\left(\mathcal{I}^{\prime}\right)\right)=\operatorname{Th}_{\Sigma}(\mathcal{I})$. Then, by Lemma 10, we have that $\langle F, \alpha\rangle: \mathcal{I}\rangle-^{s} \mathcal{I}^{\prime}$. Thus, it suffices to show that, for all $\sum \in|\mathbf{S i g n}|, \Phi \bigcup\{\phi\} \subseteq \operatorname{SEN}\left(\sum\right), \alpha_{\Sigma}(\phi) \in C_{F(\Sigma)}^{\prime}\left(\alpha_{\Sigma}(\Phi)\right)$ implies $\phi \in C_{\Sigma}(\Phi)$. Suppose, to this end that $\alpha_{\Sigma}(\phi) \in C_{F(\Sigma)}^{\prime}\left(\alpha_{\Sigma}(\Phi)\right)$ and let $T$ be a $\sum$-theory, such that $\Phi \subseteq T$. Then, there exists an $F\left(\sum\right)$-theory $T^{\prime}$, such that $T=\alpha_{\Sigma}^{-1}\left(T^{\prime}\right)$, whence $\Phi \subseteq \alpha_{\Sigma}^{-1}\left(T^{\prime}\right)$, which yields $\alpha_{\Sigma}(\Phi) \subseteq T^{\prime}$. Therefore, by our hypothesis, $\alpha_{\Sigma}(\phi) \in T^{\prime}$, But this gives $\phi \in \alpha_{\Sigma}^{-1}\left(T^{\prime}\right)$, i.e., $\phi \in T$. Since this holds for every $\sum$-theory $T$, such that $\Phi \subseteq T$, we must have $\phi \in C_{\Sigma}(\Phi)$.

Corollary 13. Let $\mathcal{I}=\left\langle\right.$ Sign, SEN, $\left.\left\{C_{\sum}\right\}_{\sum \in|\operatorname{Sign}|}\right\rangle$ and $\mathcal{I}^{\prime}=\left\langle\mathbf{S i g n}^{\prime}\right.$, $\left.\mathrm{SEN}^{\prime},\left\{C_{\sum}^{\prime}\right\}_{\sum \in\left|\operatorname{Sign}^{\prime}\right|}\right\rangle$ be $\pi$-institutions, $N, N^{\prime}$ be categories of natural transformations on SEN and $\mathrm{SEN}^{\prime}$, respectively, and $\langle F, \alpha\rangle: \mathcal{I} \rightarrow^{s} \mathcal{I}^{\prime}$ an $\left(N, N^{\prime}\right)$-translation. $\langle F, \alpha\rangle$ is an $\left(N, N^{\prime}\right)$-strong logical morphism if and only if, for all $\sum \in|\mathbf{S i g n}|,\left\{\alpha_{\Sigma}^{-1}\left(T^{\prime}\right): T^{\prime}\right.$ is an $F\left(\sum\right)$-theory $\}$ is the set of all $\sum$-theories of $\mathcal{I}$, i.e., $\operatorname{Th}_{\Sigma}(\mathcal{I})=\alpha_{\Sigma}^{-1}\left(\operatorname{Th}_{F(\Sigma)}\left(\mathcal{I}^{\prime}\right)\right)$.

Using similar conditions, bilogical and strong bilogical morphisms may be characterized as follows:

Lemma 14. Suppose $\mathcal{I}=\left\langle\right.$ Sign, SEN, $\left.\left\{C_{\sum}\right\}_{\sum \in \mid \text { Sign } \mid}\right\rangle, \mathcal{I}^{\prime}=\left\langle\right.$ Sign' $^{\prime}$, $\left.\mathrm{SEN}^{\prime},\left\{C_{\sum}^{\prime}\right\}_{\sum \in\left|\mathbf{S i g n}^{\prime}\right|}\right\rangle$ are $\pi$-institutions. A surjective singleton translation $\langle F, \alpha\rangle: \mathcal{I} \rightarrow^{s} \mathcal{I}^{\prime}$ is a surjective singleton interpretation $\langle F, \alpha\rangle: \mathcal{I} \vdash^{s} \mathcal{I}^{\prime}$ iff, for all $\sum \in|\operatorname{Sign}|, \phi \in \operatorname{SEN}(\Sigma)$,

$$
\alpha_{\Sigma}^{-1}\left(C_{F(\Sigma)}^{\prime}\left(\alpha_{\Sigma}(\phi)\right)\right)=C_{\Sigma}(\phi) .
$$


Proof. Suppose that $\langle F, \alpha\rangle: \mathcal{I} \vdash^{s} \mathcal{I}^{\prime}$ is surjective. Then, we have, for all $\sum \in|\mathbf{S i g n}|, \phi, \psi \in \operatorname{SEN}(\Sigma)$,

$$
\begin{aligned}
\psi \in C_{\Sigma}(\phi) \quad \text { iff } & \alpha_{\Sigma}(\psi) \in C_{F(\Sigma)}^{\prime}\left(\alpha_{\Sigma}(\phi)\right) \\
& \text { iff } \quad \psi \in \alpha_{\Sigma}^{-1}\left(C_{F(\Sigma)}^{\prime}\left(\alpha_{\Sigma}(\phi)\right)\right),
\end{aligned}
$$

whence $\alpha_{\Sigma}^{-1}\left(C_{F(\Sigma)}^{\prime}\left(\alpha_{\Sigma}(\phi)\right)\right)=C_{\Sigma}(\phi)$.

Suppose, conversely, that the given condition holds. Then, for all $\sum \in|\operatorname{Sign}|, \Phi \cup\{\phi\} \subseteq \operatorname{SEN}(\Sigma)$,

$$
\begin{array}{rll}
\phi \in C_{\Sigma}(\Phi) \quad \text { iff } & \phi \in \alpha_{\Sigma}^{-1}\left(C_{F(\Sigma)}^{\prime}\left(\alpha_{\Sigma}(\Phi)\right)\right) \\
& \text { iff } \quad \alpha_{\Sigma}(\phi) \in C_{F(\Sigma)}^{\prime}\left(\alpha_{\Sigma}(\Phi)\right),
\end{array}
$$

whence $\langle F, \alpha\rangle: \mathcal{I} \vdash^{s} \mathcal{I}^{\prime}$ is a surjective singleton interpretation.

Corollary 15. Let $\mathcal{I}=\left\langle\operatorname{Sign}, \mathrm{SEN},\left\{C_{\sum}\right\}_{\sum \in|\operatorname{Sign}|}\right\rangle$ and $\mathcal{I}^{\prime}=\left\langle\mathbf{S i g n}^{\prime}\right.$, $\left.\mathrm{SEN}^{\prime},\left\{C_{\sum}^{\prime}\right\}_{\sum \in\left|\mathbf{S i g n}^{\prime}\right|}\right\rangle$ be $\pi$-institutions, with $N, N^{\prime}$ categories of natural transformations on SEN and $\mathrm{SEN}^{\prime}$, respectively. A surjective $\left(N, N^{\prime}\right)$ translation $\langle F, \alpha\rangle: \mathcal{I} \rightarrow^{s} \mathcal{I}^{\prime}$ is an $\left(N, N^{\prime}\right)$-bilogical morphism $\langle F, \alpha\rangle$ : $\mathcal{I} \vdash^{s} \mathcal{I}^{\prime}$ iff, for all $\sum \in|\operatorname{Sign}|, \phi \in \operatorname{SEN}\left(\sum\right)$,

$$
\alpha_{\Sigma}^{-1}\left(C_{F(\Sigma)}^{\prime}\left(\alpha_{\Sigma}(\phi)\right)\right)=C_{\Sigma}(\phi)
$$

Corollary 16. Let $\mathcal{I}=\left\langle\operatorname{Sign}, \operatorname{SEN},\left\{C_{\sum}\right\}_{\sum \in|\operatorname{Sign}|}\right\rangle$ and $\mathcal{I}^{\prime}=\left\langle\mathbf{S i g n}^{\prime}\right.$, $\left.\mathrm{SEN}^{\prime},\left\{C_{\sum}^{\prime}\right\}_{\sum \in\left|\mathbf{S i g n}^{\prime}\right|}\right\rangle$ be $\pi$-institutions, with $N, N^{\prime}$ categories of natural transformations on $\mathrm{SEN}$ and $\mathrm{SEN}^{\prime}$, respectively. Let $\langle F, \alpha\rangle: \mathcal{I} \vdash^{s} \mathcal{I}^{\prime}$ be an $\left(N, N^{\prime}\right)$-bilogical morphism. Then, for all $\sum \in|\operatorname{Sign}|, \Phi \subseteq \operatorname{SEN}(\Sigma)$,

$$
\alpha_{\Sigma}\left(C_{\Sigma}(\Phi)\right)=C_{F(\Sigma)}^{\prime}\left(\alpha_{\Sigma}(\Phi)\right) \text {. }
$$


Proof. Immediate by applying Lemma 14.

Next, it is shown that bilogical morphisms induce bijections between the classes of theories of the $\pi$-institutions they relate. First, a weaker result is proven for surjective singleton interpretations.

Lemma 17. Suppose $\mathcal{I}=\left\langle\right.$ Sign, SEN, $\left.\left\{C_{\sum}\right\}_{\sum \in \mid \text { Sign } \mid}\right\rangle, \mathcal{I}^{\prime}=\left\langle\right.$ Sign' $^{\prime}$ $\left.\mathrm{SEN}^{\prime},\left\{C_{\sum}^{\prime}\right\}_{\sum \in\left|\mathbf{S i g n}^{\prime}\right|}\right\rangle$ are $\pi$-institutions. Every surjective singleton interpretation $\langle F, \alpha\rangle: \mathcal{I} \vdash^{s} \mathcal{I}^{\prime}$ induces a bijection $\left|\operatorname{Th}_{\Sigma}(\mathcal{I})\right| \cong\left|\operatorname{Th}_{F(\Sigma)}\left(\mathcal{I}^{\prime}\right)\right|$, for every $\sum \in|\operatorname{Sign}|$. Moreover, this bijection is functorial in the sense that, for all $\sum \in|\mathbf{S i g n}|$ and all $\sum$-theories $T, F^{\#}\left(\left\langle\sum, T\right\rangle\right)=\langle F(\Sigma)$, $\left.C_{F(\Sigma)}^{\prime}\left(\alpha_{\Sigma}(T)\right)\right\rangle$, and $F^{\#}(f)=F(f)$, for all $f:\left\langle\Sigma_{1}, T_{1}\right\rangle \rightarrow\left\langle\Sigma_{2}, T_{2}\right\rangle$, is a functor.

Proof. Consider a theory $T \in\left|\mathbf{T h}_{\Sigma}(\mathcal{I})\right|$. Then, by Lemma 14, we have $\alpha_{\Sigma}(T) \in\left|\mathbf{T h}_{F(\Sigma)}\left(\mathcal{I}^{\prime}\right)\right|$. Thus $\alpha_{\Sigma}:\left|\mathbf{T h}_{\Sigma}(\mathcal{I})\right| \rightarrow\left|\mathbf{T h}_{F(\Sigma)}\left(\mathcal{I}^{\prime}\right)\right|$ is a well-defined mapping from the set of all $\sum$-theories of $\mathcal{I}$ to the set of all $F(\Sigma)$-theories of $\mathcal{I}^{\prime}$. It is an onto mapping since, by Lemma 14, we get $\alpha_{\Sigma}\left(\alpha_{\Sigma}^{-1}(T)\right)=T$, for all $T \in\left|\mathbf{T h}_{\Sigma}\left(\mathcal{I}^{\prime}\right)\right|$, and, by Lemma $12, \alpha_{\Sigma}^{-1}(T)$ is a $F(\Sigma)$-theory of $\mathcal{I}$. Moreover, it is one-one, since, for all $\sum$-theories $T_{1}, T_{2}$, $\alpha_{\Sigma}\left(T_{1}\right)=\alpha_{\Sigma}\left(T_{2}\right) \quad$ implies $\quad \alpha_{\Sigma}^{-1}\left(C_{F(\Sigma)}^{\prime}\left(\alpha_{\Sigma}\left(T_{1}\right)\right)\right)=\alpha_{\Sigma}^{-1}\left(C_{F(\Sigma)}^{\prime}\left(\alpha_{\Sigma}\left(T_{2}\right)\right)\right)$, whence, by Lemma 14 , we get $C_{\Sigma}\left(T_{1}\right)=C_{\Sigma}\left(T_{2}\right)$, i.e., $T_{1}=T_{2}$.

That $F^{\#}$ is functorial follows directly from Theorem 8.1 (i) of [21].

Lemma 17 has the following immediate corollary when translated to bilogical morphisms.

Corollary 18. Let $\mathcal{I}=\left\langle\right.$ Sign, $\left.\operatorname{SEN},\left\{C_{\sum}\right\}_{\sum \in|\operatorname{Sign}|}\right\rangle$ and $\mathcal{I}=\left\langle\right.$ Sign'$^{\prime}$ $\left.\mathrm{SEN}^{\prime},\left\{C_{\sum}^{\prime}\right\}_{\sum \in\left|\mathbf{S i g n}^{\prime}\right|}\right\rangle$ be $\pi$-institutions, with $N, N^{\prime}$ categories of natural transformations on SEN and $\mathrm{SEN}^{\prime}$, respectively. An $\left(N, N^{\prime}\right)$-bilogical 
morphism $\langle F, \alpha\rangle: \mathcal{I} \vdash^{s} \mathcal{I}^{\prime}$ induces a bijection $\left|\mathbf{T h}_{\Sigma}(\mathcal{I})\right| \cong\left|\mathbf{T h}_{F(\Sigma)}\left(\mathcal{I}^{\prime}\right)\right|$, for every $\sum \in|\mathbf{S i g n}|$. Moreover, this bijection is functorial in the sense that, for all $\sum \in|\mathbf{S i g n}|$ and all $\sum$-theories $T, F^{\#}\left(\left\langle\sum, T\right\rangle\right)=\langle F(\Sigma)$, $\left.C_{F(\Sigma)}^{\prime}\left(\alpha_{\Sigma}(T)\right)\right\rangle$, and $F^{\#}(f)=F(f)$, for all $f:\left\langle\Sigma_{1}, T_{1}\right\rangle \rightarrow\left\langle\Sigma_{2}, T_{2}\right\rangle$, is a functor.

The following result asserts that surjective singleton interpretations with isomorphic signature functors give rise to isomorphisms between categories of theories.

Lemma 19. Suppose $\mathcal{I}=\left\langle\right.$ Sign, $\left.\mathrm{SEN},\left\{C_{\sum}\right\}_{\sum \in|\operatorname{Sign}|}\right\rangle, \mathcal{I}^{\prime}=\left\langle\mathbf{S i g n}^{\prime}, \mathrm{SEN}^{\prime}\right.$, $\left.\left\{C_{\sum}^{\prime}\right\}_{\sum \in\left|\mathbf{S i g n}^{\prime}\right|}\right\rangle$ are $\pi$-institutions and $\langle F, \alpha\rangle: \mathcal{I}^{s} \mathcal{I}^{\prime}$ is a surjective singleton interpretation, such that $F$ is an isomorphism. Then $G^{\#}\left(\left\langle\Sigma^{\prime}, T^{\prime}\right\rangle\right)=\left\langle F^{-1}\left(\Sigma^{\prime}\right), \alpha_{\Sigma}^{-1}\left(T^{\prime}\right)\right\rangle$, for all $\Sigma^{\prime} \in\left|\mathbf{S i g n}^{\prime}\right|$ and $\Sigma^{\prime}$-theories $T^{\prime}$, and $G^{\#}(f)=F^{-1}(f)$, for all $f:\left\langle\sum_{1}^{\prime}, T_{1}^{\prime}\right\rangle \rightarrow\left\langle\Sigma_{2}^{\prime}, T_{2}^{\prime}\right\rangle$, is a functor and $G^{\#}$ and $F^{\#}$ establish an isomorphism between the categories of theories of $\mathcal{I}$ and $\mathcal{I}^{\prime}$.

Proof. It suffices to show that, for all $f^{\prime}:\left\langle\Sigma_{1}^{\prime}, T_{1}^{\prime}\right\rangle \rightarrow\left\langle\Sigma_{2}^{\prime}, T_{2}^{\prime}\right\rangle$ in the category of theories of $\mathcal{I}^{\prime}$, the mapping $F^{-1}\left(f^{\prime}\right): F^{-1}\left(\Sigma_{1}^{\prime}\right) \rightarrow F^{-1}\left(\Sigma_{2}^{\prime}\right)$ in Sign is a well-defined theory morphism $F^{-1}\left(f^{\prime}\right):\left\langle F^{-1}\left(\Sigma_{1}^{\prime}\right)\right.$, $\alpha_{F^{-1}\left(\Sigma_{1}^{\prime}\right)}^{-1}\left(T_{1}^{\prime}\right) \rightarrow\left\langle F^{-1}\left(\Sigma_{2}^{\prime}\right), \alpha_{F^{-1}\left(\Sigma_{2}^{\prime}\right)}^{-1}\left(T_{2}^{\prime}\right)\right\rangle$, i.e., that, if $\operatorname{SEN}^{\prime}\left(f^{\prime}\right)\left(T_{1}^{\prime}\right) \subseteq T_{2}^{\prime}$, then

$$
\operatorname{SEN}\left(F^{-1}\left(f^{\prime}\right)\right)\left(\alpha_{F^{-1}\left(\Sigma_{1}^{\prime}\right)}^{-1}\left(T_{1}^{\prime}\right)\right) \subseteq \alpha_{F^{-1}\left(\Sigma_{2}^{\prime}\right)}^{-1}\left(T_{2}^{\prime}\right)
$$

We in fact show that, for all $\Sigma_{1}^{\prime}, \Sigma_{2}^{\prime} \in\left|\operatorname{Sign}^{\prime}\right|, f^{\prime} \in \operatorname{Sign}^{\prime}\left(\Sigma_{1}^{\prime}, \Sigma_{2}^{\prime}\right)$, and $\psi \in \operatorname{SEN}^{\prime}\left(\Sigma_{1}^{\prime}\right)$, 


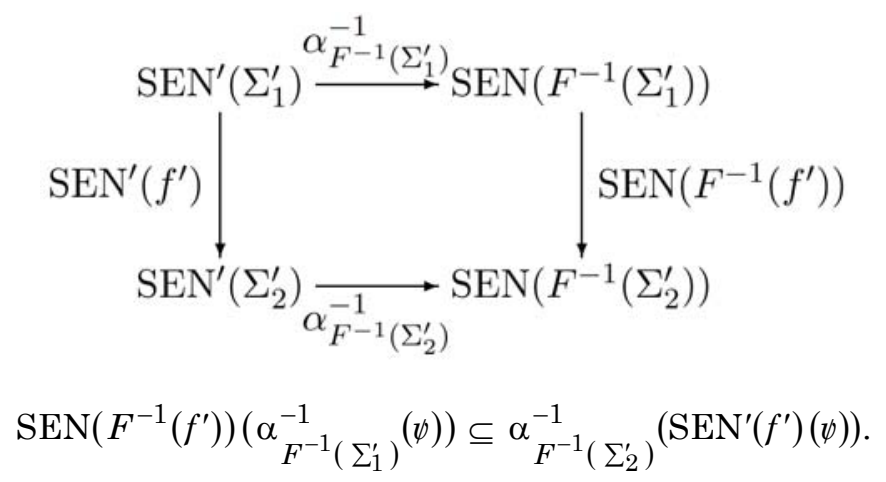

We have

$$
\begin{gathered}
\phi \in \operatorname{SEN}\left(F^{-1}\left(f^{\prime}\right)\right)\left(\alpha_{F^{-1}\left(\Sigma_{1}^{\prime}\right)}^{-1}(\psi)\right) \\
\text { implies } \alpha_{F^{-1}\left(\Sigma_{2}^{\prime}\right)}(\phi) \in \alpha_{F^{-1}\left(\Sigma_{2}^{\prime}\right)}\left(\operatorname{SEN}\left(F^{-1}\left(f^{\prime}\right)\right)\left(\alpha_{F^{-1}\left(\Sigma_{1}^{\prime}\right)}^{-1}(\psi)\right)\right) \\
\text { implies } \alpha_{F^{-1}\left(\Sigma_{2}^{\prime}\right)}(\phi) \in \operatorname{SEN}^{\prime}\left(f^{\prime}\right)\left(\alpha_{F^{-1}\left(\Sigma_{1}^{\prime}\right)}\left(\alpha_{F^{-1}\left(\Sigma_{1}^{\prime}\right)}^{-1}(\psi)\right)\right) \\
\text { implies } \alpha_{F^{-1}\left(\Sigma_{2}^{\prime}\right)}(\phi) \in \operatorname{SEN}^{\prime}\left(f^{\prime}\right)(\psi) \\
\text { implies } \phi \in \alpha_{F^{-1}\left(\Sigma_{2}^{\prime}\right)}\left(\operatorname{SEN}^{\prime}\left(f^{\prime}\right)(\psi)\right)
\end{gathered}
$$

Finally, $F^{\#}$ and $G^{\#}$ are inverses of each other by Lemma 17.

The following theorem now follows by combining Lemmas 17 and 19 . provides an analogue of Propositions 1.4 and 1.5 of [11].

Theorem 20. Let $\mathcal{I}=\left\langle\operatorname{Sign}, \mathrm{SEN},\left\{C_{\sum}\right\}_{\sum \in|\mathrm{Sign}|}\right\rangle$ and $\mathcal{I}^{\prime}=\left\langle\right.$ Sign' $^{\prime}, \mathrm{SEN}^{\prime}$, $\left.\left\{C_{\sum}^{\prime}\right\}_{\sum \in\left|\mathbf{S i g n}^{\prime}\right|}\right\rangle$ be $\pi$-institutions and $\langle F, \alpha\rangle: \mathcal{I} \vdash^{s} \mathcal{I}^{\prime}$ be a surjective singleton interpretation, such that $F$ is an isomorphism. Then the categories of theories of $\mathcal{I}$ and $\mathcal{I}^{\prime}$ are isomorphic categories via the isomorphisms

$$
F^{\#}\left(\left\langle\sum, T\right\rangle\right)=\left\langle F(\Sigma), C_{F(\Sigma)}^{\prime}\left(\alpha_{\Sigma}(T)\right)\right\rangle
$$




$$
G^{\#}\left(\left\langle\Sigma^{\prime}, T^{\prime}\right\rangle\right)=\left\langle F^{-1}\left(\Sigma^{\prime}\right), \alpha_{\Sigma}^{-1}\left(T^{\prime}\right)\right\rangle,
$$

for all $\Sigma \in|\mathbf{S i g n}|, \Sigma^{\prime} \in\left|\mathbf{S i g n}^{\prime}\right|$, and all $\sum$-theories $T$ and $\Sigma^{\prime}$-theories $T^{\prime}$.

The last result of the section relates $\left(N, N^{\prime}\right)$-bilogical morphisms between two $\pi$-institutions with the Tarski $N$-congruence system of the first and the Tarski $N^{\prime}$-congruence system of the second. Namely, it is shown that applying the inverse of an $\left(N, N^{\prime}\right)$-bilogical morphism between $\mathcal{I}$ and $\mathcal{I}^{\prime}$ carries the Tarski $N^{\prime}$-congruence system of $\mathcal{I}^{\prime}$ to the Tarski $N$-congruence system of $\mathcal{I}$. This is the analogue of Proposition 1.7 of [11].

Theorem 21. Let $\mathcal{I}=\left\langle\right.$ Sign, SEN, $\left.\left\{C_{\sum}\right\}_{\sum \in|\operatorname{Sign}|}\right\rangle$ and $\mathcal{I}^{\prime}=\left\langle\mathbf{S i g n}^{\prime}\right.$, $\left.\mathrm{SEN}^{\prime},\left\{C_{\Sigma}^{\prime}\right\}_{\Sigma \in\left|\mathbf{S i g n}^{\prime}\right|}\right\rangle$ be $\pi$-institutions and $N, N^{\prime}$ be categories of natural transformations on SEN, SEN', respectively. Let $\langle F, \alpha\rangle: \mathcal{I} \vdash^{s} \mathcal{I}^{\prime}$ be an $\left(N, N^{\prime}\right)$-bilogical morphism. Then

$$
\widetilde{\Omega}_{\Sigma}^{N}(\mathcal{I})=\alpha_{\Sigma}^{-1}\left(\widetilde{\Omega}_{F(\Sigma)}^{N^{\prime}}\left(\mathcal{I}^{\prime}\right)\right), \text { for all } \Sigma \in|\mathbf{S i g n}| .
$$

Proof. We have $\langle\phi, \psi\rangle \in \alpha_{\Sigma}^{-1}\left(\widetilde{\Omega}_{F(\Sigma)}^{N^{\prime}}\left(\mathcal{I}^{\prime}\right)\right)$ iff $\left\langle\alpha_{\Sigma}(\phi), \alpha_{\Sigma}(\psi)\right) \in \widetilde{\Omega}_{F(\Sigma)}^{N^{\prime}}\left(\mathcal{I}^{\prime}\right)$ iff, by Theorem 4, for all $\Sigma^{\prime} \in|\operatorname{Sign}|, f \in \operatorname{Sign}\left(\sum, \Sigma^{\prime}\right), \tau^{\prime}: \mathrm{SEN}^{\prime k} \rightarrow \mathrm{SEN}^{\prime}$ and all $\vec{\chi}^{\prime} \in \operatorname{SEN}^{\prime}\left(F\left(\Sigma^{\prime}\right)\right)^{k}$,

$$
\begin{aligned}
& C_{F\left(\Sigma^{\prime}\right)}^{\prime}\left(\tau_{F\left(\Sigma^{\prime}\right)}^{\prime}\left(\operatorname{SEN}^{\prime}(F(f))\left(\alpha_{\Sigma}(\phi)\right), \vec{\chi}^{\prime}\right)\right) \\
& \quad=C_{F\left(\Sigma^{\prime}\right)}^{\prime}\left(\tau^{\prime} F\left(\Sigma^{\prime}\right)\left(\operatorname{SEN}^{\prime}(F(f))\left(\alpha_{\Sigma}(\psi)\right), \vec{\chi}^{\prime}\right)\right),
\end{aligned}
$$

iff, for all $\Sigma^{\prime} \in|\operatorname{Sign}|, f \in \operatorname{Sign}\left(\sum, \Sigma^{\prime}\right), \tau^{\prime}: \mathrm{SEN}^{\prime k} \rightarrow \mathrm{SEN}^{\prime}$ and all $\vec{\chi}^{\prime} \in \operatorname{SEN}^{\prime}\left(F\left(\Sigma^{\prime}\right)\right)^{k}$,

$$
C_{F\left(\Sigma^{\prime}\right)}^{\prime}\left(\tau_{F\left(\Sigma^{\prime}\right)}^{\prime}\left(\alpha_{\Sigma^{\prime}}\left(\operatorname{SEN}(f(\phi)), \vec{\chi}^{\prime}\right)\right)=C_{F\left(\Sigma^{\prime}\right)}^{\prime}\left(\tau_{F\left(\Sigma^{\prime}\right)}^{\prime}\left(\alpha_{\Sigma^{\prime}}(\operatorname{SEN}(f)(\psi)), \vec{\chi}^{\prime}\right)\right),\right.
$$

iff, for all $\Sigma^{\prime} \in|\operatorname{Sign}|, f \in \operatorname{Sign}\left(\sum, \Sigma^{\prime}\right), \tau^{\prime}: \operatorname{SEN}^{\prime} \rightarrow \operatorname{SEN}^{\prime}, \vec{\chi} \in \operatorname{SEN}\left(\Sigma^{\prime}\right)^{k}$, 


$$
C_{F\left(\Sigma^{\prime}\right)}^{\prime}\left(\tau_{F\left(\Sigma^{\prime}\right)}^{\prime}\left(\alpha_{\Sigma^{\prime}}^{k}(\operatorname{SEN}(f)(\phi), \vec{\chi})\right)\right)=C_{F\left(\Sigma^{\prime}\right)}^{\prime}\left(\tau_{F\left(\Sigma^{\prime}\right)}^{\prime}\left(\alpha_{\Sigma^{\prime}}^{k}(\operatorname{SEN}(f)(\psi), \vec{\chi})\right)\right),
$$

iff, for all $\Sigma^{\prime} \in|\operatorname{Sign}|, f \in \operatorname{Sign}\left(\sum, \Sigma^{\prime}\right), \tau: \operatorname{SEN}^{k} \rightarrow \operatorname{SEN}, \vec{\chi} \in \operatorname{SEN}\left(\Sigma^{\prime}\right)^{k}$,

$$
C_{F\left(\Sigma^{\prime}\right)}^{\prime}\left(\alpha_{\Sigma^{\prime}}\left(\tau_{\Sigma^{\prime}}(\operatorname{SEN}(f)(\phi), \vec{\chi})\right)\right)=C_{F\left(\Sigma^{\prime}\right)}^{\prime}\left(\alpha_{\Sigma^{\prime}}\left(\tau_{\Sigma^{\prime}}(\operatorname{SEN}(f)(\psi), \vec{\chi})\right)\right),
$$

iff, for all $\Sigma^{\prime} \in|\operatorname{Sign}|, f \in \operatorname{Sign}\left(\sum, \Sigma^{\prime}\right), \tau: \operatorname{SEN}^{k} \rightarrow \operatorname{SEN}, \vec{\chi} \in \operatorname{SEN}\left(\Sigma^{\prime}\right)^{k}$,

$$
C_{\Sigma^{\prime}}\left(\tau_{\Sigma^{\prime}}(\operatorname{SEN}(f)(\phi), \vec{\chi})\right)=C_{\Sigma^{\prime}}\left(\tau_{\Sigma^{\prime}}(\operatorname{SEN}(f)(\psi), \vec{\chi})\right),
$$

iff, again by Theorem $4,\langle\phi, \psi\rangle \in \widetilde{\Omega}_{\Sigma}^{N}(\mathcal{I})$.

\section{Logical Quotients}

Let $\mathcal{I}=\left\langle\right.$ Sign, SEN, $\left.\left\{C_{\sum}\right\}_{\sum \in \mid \text { Sign } \mid}\right\rangle$ be a $\pi$-institution and $\theta$ be a logical equivalence system of $\mathcal{I}$. Define the triple $\mathcal{I} / \theta=\left\langle\operatorname{Sign}, \mathrm{SEN}^{\theta}\right.$, $\left.\left\{C_{\Sigma}^{\theta}\right\}_{\Sigma \in|\mathbf{S i g n}|}\right\rangle$ as follows:

- $\operatorname{SEN}^{\theta}: \operatorname{Sign} \rightarrow$ Set is defined by $\operatorname{SEN}^{\theta}(\Sigma)=\operatorname{SEN}(\Sigma) / \theta_{\Sigma}$, for all $\sum \in|\operatorname{Sign}|$, and, given $\Sigma_{1}, \Sigma_{2} \in|\operatorname{Sign}|, f \in \operatorname{Sign}\left(\Sigma_{1}, \Sigma_{2}\right), \phi \in \operatorname{SEN}\left(\Sigma_{1}\right)$,

$$
\operatorname{SEN}^{\theta}(f)\left(\phi / \theta_{\Sigma_{1}}\right)=\operatorname{SEN}(f)(\phi) / \theta_{\Sigma_{2}} .
$$

- For all $\Sigma \in|\operatorname{Sign}|, \Phi \bigcup\{\psi\} \subseteq \operatorname{SEN}(\Sigma)$,

$$
\psi / \theta_{\Sigma} \in C_{\Sigma}^{\theta}\left(\Phi / \theta_{\Sigma}\right) \quad \text { iff } \quad \psi / \theta_{\Sigma} \subseteq C_{\Sigma}\left(\bigcup_{\phi \in \Phi} \phi / \theta_{\Sigma}\right)
$$

The next proposition asserts that $\mathcal{I} / \theta$ is also a $\pi$-institution.

Proposition 22. Given a r-institution $\mathcal{I}=\langle$ Sign, SEN, $C\rangle$ and $a$ logical equivalence system $\theta$ of $\mathcal{I}, \mathcal{I} / \theta=\left\langle\operatorname{Sign}, \operatorname{SEN}^{\theta}, C^{\theta}\right\rangle$ is also a $\pi$-institution. 
Proof. The sentence functor is well-defined at the morphism level, since, by the definition of an equivalence system, for all $\Sigma_{1}, \Sigma_{2} \in|\mathbf{S i g n}|$, and all $f \in \operatorname{Sign}\left(\Sigma_{1}, \Sigma_{2}\right)$, we have $\operatorname{SEN}(f)\left(\theta_{\Sigma_{1}}\right) \subseteq \theta_{\Sigma_{2}}$. Also the closure $C_{\Sigma}^{\theta}$ is well-defined, for all $\Sigma \in|\operatorname{Sign}|$. To see this, let $\Sigma \in|\operatorname{Sign}|$, $\Phi \bigcup \Phi^{\prime} \cup\left\{\psi, \psi^{\prime}\right\} \subseteq \operatorname{SEN}(\Sigma)$, such that $\left\langle\psi, \psi^{\prime}\right\rangle \in \theta_{\Sigma}$ and $\Phi / \theta_{\Sigma}=\Phi^{\prime} / \theta_{\Sigma}$. Then, since $\theta$ is a logical equivalence system, we get $C_{\Sigma}(\Phi)=C_{\Sigma}\left(\Phi^{\prime}\right)$, whence

$$
\begin{aligned}
& \psi / \theta_{\Sigma} \in C_{\Sigma}^{\theta}\left(\Phi / \theta_{\Sigma}\right) \quad \text { iff } \quad \psi / \theta_{\Sigma} \subseteq C_{\Sigma}\left(\bigcup \Phi / \theta_{\Sigma}\right) \\
& \text { iff } \quad \phi^{\prime} / \theta_{\Sigma} \subseteq C_{\Sigma}\left(\bigcup \Phi^{\prime} / \theta_{\Sigma}\right) \\
& \text { iff } \quad \psi^{\prime} / \theta_{\Sigma} \in C_{\Sigma}^{\theta}\left(\Phi^{\prime} / \theta_{\Sigma}\right) \text {. }
\end{aligned}
$$

It only remains to verify the four conditions of a closure system for $C^{\theta}$. Conditions (a) and (c) are straightforward. So, only (b) and (d) will be verified in detail.

For (b), suppose that $\psi / \theta_{\Sigma} \in C_{\Sigma}^{\theta}\left(C_{\Sigma}^{\theta}\left(\Phi / \theta_{\Sigma}\right)\right)$. Then we get

$$
\psi / \theta_{\Sigma} \in C_{\Sigma}^{\theta}\left(\left\{\chi / \theta_{\Sigma}: \chi / \theta_{\Sigma} \subseteq C_{\Sigma}\left(\bigcup \Phi / \theta_{\Sigma}\right)\right\}\right)
$$

whence $\quad \psi / \theta_{\Sigma} \subseteq C_{\Sigma}\left(\bigcup\left\{\chi / \theta_{\Sigma}: \chi / \theta_{\Sigma} \subseteq C_{\Sigma}\left(\bigcup \Phi / \theta_{\Sigma}\right)\right\}\right)$. Therefore, $\psi / \theta_{\Sigma} \subseteq C_{\Sigma}\left(\bigcup \Phi / \theta_{\Sigma}\right)$, i.e., $\psi / \theta_{\Sigma} \in C_{\Sigma}^{\theta}\left(\Phi / \theta_{\Sigma}\right)$.

Finally, for (d), suppose that $\Sigma_{1}, \Sigma_{2} \in|\operatorname{Sign}|, f \in \operatorname{Sign}\left(\Sigma_{1}, \Sigma_{2}\right)$. Then, if $\Phi \subseteq \operatorname{SEN}\left(\Sigma_{1}\right)$, we need to show

$$
\operatorname{SEN}^{\theta}(f)\left(C_{\Sigma_{1}}^{\theta}\left(\Phi / \theta_{\Sigma_{1}}\right)\right) \subseteq C_{\Sigma_{2}}^{\theta}\left(\operatorname{SEN}^{\theta}(f)\left(\Phi / \theta_{\Sigma_{1}}\right)\right)
$$

Suppose, to this end, that $\psi / \theta_{\Sigma_{1}} \in C_{\Sigma_{1}}^{\theta}\left(\Phi / \theta_{\Sigma_{1}}\right)$. Then, we have $\psi / \theta_{\Sigma_{1}}$ $\subseteq C_{\Sigma_{1}}\left(\bigcup \Phi / \theta_{\Sigma_{1}}\right)$. Therefore $\operatorname{SEN}(f)\left(\psi / \theta_{\Sigma_{1}}\right) \subseteq C_{\Sigma_{2}}\left(\operatorname{SEN}(f)\left(\bigcup \Phi / \theta_{\Sigma_{1}}\right)\right)$. But, for all $\psi^{\prime} \in \psi / \theta_{\Sigma_{1}}$, we have $\operatorname{SEN}(f)\left(\psi^{\prime}\right) \in \operatorname{SEN}(f)(\psi) / \theta_{\Sigma_{2}}$, whence, since $\theta$ is logical, 


$$
C_{\Sigma_{2}}\left(\operatorname{SEN}(f)\left(\psi / \theta_{\Sigma_{1}}\right)\right)=C_{\Sigma_{2}}\left(\operatorname{SEN}(f)\left(\psi^{\prime}\right) / \theta_{\Sigma_{2}}\right)
$$

and, similarly, $\quad C_{\Sigma_{2}}\left(\operatorname{SEN}(f)\left(\bigcup \Phi / \theta_{\Sigma_{1}}\right)\right)=C_{\Sigma_{2}}\left(\bigcup \operatorname{SEN}(f)(\Phi) / \theta_{\Sigma_{2}}\right)$. Therefore, we obtain $\operatorname{SEN}(f)(\psi) / \theta_{\Sigma_{2}} \subseteq C_{\Sigma_{2}}\left(\bigcup \operatorname{SEN}(f)(\Phi) / \theta_{\Sigma_{2}}\right)$, i.e.,

$$
\operatorname{SEN}^{\theta}(f)\left(\psi / \theta_{\Sigma_{1}}\right) \in C_{\Sigma_{2}}^{\theta}\left(\operatorname{SEN}^{\theta}(f)\left(\Phi / \theta_{\Sigma_{1}}\right)\right)
$$

Given a $\pi$-institution $\mathcal{I}$ and a logical equivalence system $\theta$ of $\mathcal{I}$, the $\pi$-institution $\mathcal{I} / \theta$ will be called the logical quotient of $\mathcal{I}$ by the logical equivalence system $\theta$.

Now consider a $\pi$-institution $\mathcal{I}$, a logical equivalence system $\theta$ of $\mathcal{I}$, and the logical quotient $\mathcal{I} / \theta$ of $\mathcal{I}$ by $\theta$. Define $\left\langle I_{\text {Sign }}, \pi^{\theta}\right\rangle: \mathcal{I} \rightarrow{ }^{s} \mathcal{I} / \theta$, by

$$
\pi_{\Sigma}^{\theta}(\phi)=\phi / \theta_{\Sigma}, \quad \text { for all } \Sigma \in|\operatorname{Sign}|, \phi \in \operatorname{SEN}(\Sigma)
$$

The pair $\left\langle I_{\text {Sign }}, \pi^{\theta}\right\rangle$ is a singleton translation from $\mathcal{I}$ to $\mathcal{I}^{\theta}$. In fact, we have

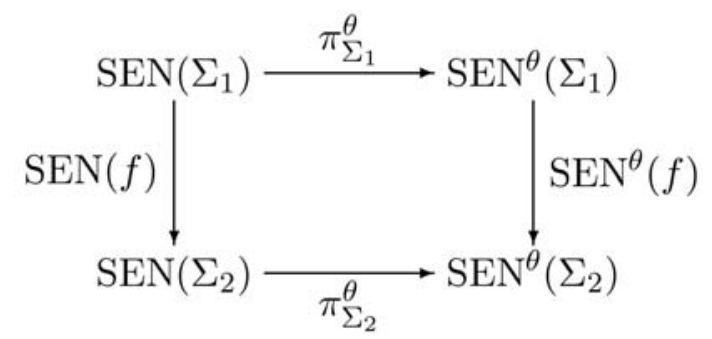

$$
\begin{aligned}
\pi_{\Sigma_{2}}^{\theta}(\operatorname{SEN}(f)(\phi)) & =\quad \operatorname{SEN}(f)(\phi) / \theta_{\Sigma_{2}} \\
& =\operatorname{SEN}^{\theta}(f)\left(\phi / \theta_{\Sigma_{1}}\right) \\
& =\operatorname{SEN}^{\theta}(f)\left(\pi_{\Sigma_{1}}^{\theta}(\phi)\right) .
\end{aligned}
$$


Let $\mathcal{I}$ be a $\pi$-institution, $N$ be a category of natural transformations on SEN, and $\theta$ be a logical $N$-congruence system of $\mathcal{I}$. Let $\sigma: \mathrm{SEN}^{k} \rightarrow \mathrm{SEN}$ be in $N$. Since $\theta$ is an $N$-congruence system on $\mathcal{I}, \sigma^{\theta}:\left(\mathrm{SEN}^{\theta}\right)^{k} \rightarrow \mathrm{SEN}^{\theta}$, given by

$$
\sigma_{\Sigma}^{\theta}\left(\vec{\phi} / \theta_{\Sigma}\right)=\sigma_{\Sigma}(\vec{\phi}) / \theta_{\Sigma}, \quad \text { for all } \sum \in|\operatorname{Sign}|, \vec{\phi} \in \operatorname{SEN}(\Sigma)^{k}
$$

where $\vec{\phi} / \theta_{\Sigma}=\left\langle\phi_{0} / \theta_{\Sigma}, \ldots, \phi_{k-1} / \theta_{\Sigma}\right\rangle$, is well-defined. Furthermore, it is a natural transformation, since, for every $\Sigma_{1}, \Sigma_{2} \in \mid$ Sign $\mid$, $f \in \operatorname{Sign}\left(\Sigma_{1}, \Sigma_{2}\right)$,

$$
\begin{aligned}
\operatorname{SEN}\left(\Sigma_{1}\right)^{k} / \theta_{\Sigma_{1}} & \stackrel{\sigma_{\Sigma_{1}}^{\theta}}{\longrightarrow} \operatorname{SEN}\left(\Sigma_{1}\right) / \theta_{\Sigma_{1}} \\
\operatorname{SEN}^{\theta}(f)^{k} \mid & \operatorname{SEN}^{\theta}(f) \\
\operatorname{SEN}\left(\Sigma_{2}\right)^{k} / \theta_{\Sigma_{2}} & \underset{\sigma_{\Sigma_{2}}^{\theta}}{\longrightarrow} \operatorname{SEN}\left(\Sigma_{2}\right) / \theta_{\Sigma_{2}} \\
\operatorname{SEN}^{\theta}(f)\left(\sigma_{\Sigma_{1}}^{\theta}\left(\vec{\phi} / \theta_{\Sigma_{1}}\right)\right)= & \operatorname{SEN}(f)\left(\sigma_{\Sigma_{1}}(\vec{\phi}) / \theta_{\Sigma_{1}}\right) \\
& =\quad \sigma_{\Sigma_{2}}\left(\operatorname{SEN}(f)^{k}(\vec{\phi})\right) / \theta_{\Sigma_{2}} \\
& =\quad \sigma_{\Sigma_{2}}^{\theta}\left(\operatorname{SEN}(f)^{k}(\vec{\phi}) / \theta_{\Sigma_{2}}\right) \\
& =\quad \sigma_{\Sigma_{2}}^{\theta}\left(\operatorname{SEN}(f)^{k}\left(\vec{\phi} / \theta_{\Sigma_{1}}\right)\right)
\end{aligned}
$$

Let $U^{\theta}$ be the category of all natural transformations on $\operatorname{SEN}^{\theta}$ that are of the form $\sigma^{\theta}$, for some $\sigma$ in $U$. Denote by $N^{\theta}$ the subcategory of the category $U^{\theta}$ consisting of the natural transformations of the form $\sigma^{\theta}$ for $\sigma$ in $N$. 
Proposition 23. Let $\mathcal{I}=\left\langle\right.$ Sign, SEN, $\left.\left\{C_{\sum}\right\}_{\sum \in|\operatorname{Sign}|}\right\rangle$ be a $\pi$-institution.

(1) If $\theta$ a logical equivalence system of $\mathcal{I}$, then $\left\langle I_{\text {Sign }}, \pi^{\theta}\right\rangle: \mathcal{I} \vdash^{s} \mathcal{I} / \theta$ is a surjective singleton interpretation from $\mathcal{I}$ to $\mathcal{I} / \theta$.

(2) If $N$ is a category of natural transformations on SEN and $\theta$ a logical $N$-congruence system of $\mathcal{I}$, then $\left\langle I_{\text {Sign }}, \pi^{\theta}\right\rangle: \mathcal{I} \vdash^{s} \mathcal{I} / \theta$ is a $\left(N, N^{\theta}\right)$-bilogical morphism from $\mathcal{I}$ to $\mathcal{I} / \theta$.

Proof. It has already been shown that $\left\langle I_{\text {Sign }}, \pi^{\theta}\right\rangle$ is a singleton translation and it is clearly surjective. Thus, for the first part, it suffices to show that it is an interpretation and for the second part that it is also an $\left(N, N^{\theta}\right)$-morphism.

First, it is shown that $\left\langle I_{\text {Sign }}, \pi^{\theta}\right\rangle: \mathcal{I} \rightarrow^{s} \mathcal{I} / \theta$ is an interpretation. To this end, suppose that $\Sigma \in|\operatorname{Sign}|, \Phi \bigcup\{\phi\} \subseteq \operatorname{SEN}(\Sigma)$. Then we have

$$
\begin{array}{rll}
\phi \in C_{\Sigma}(\Phi) \quad \text { iff } & C_{\Sigma}(\phi) \subseteq C_{\Sigma}(\Phi) \\
& \text { iff } & C_{\Sigma}\left(\phi / \theta_{\Sigma}\right) \subseteq C_{\Sigma}\left(\bigcup \Phi / \theta_{\Sigma}\right) \\
\text { iff } & \phi / \theta_{\Sigma} \subseteq C_{\Sigma}\left(\bigcup \Phi / \theta_{\Sigma}\right) \\
\text { iff } & \phi / \theta_{\Sigma} \in C_{\Sigma}^{\theta}\left(\Phi / \theta_{\Sigma}\right) .
\end{array}
$$

Finally, for all $\sigma: \operatorname{SEN}^{k} \rightarrow \operatorname{SEN}$ in $N$, and for all $\sum \in|\operatorname{Sign}|$, $\vec{\phi} \in \operatorname{SEN}(\Sigma)^{k}$

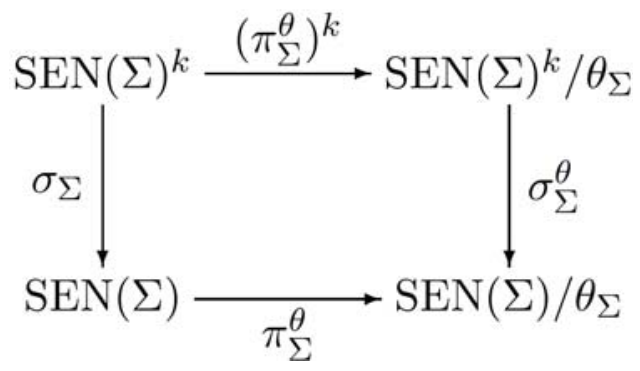




$$
\begin{aligned}
\sigma_{\Sigma}^{\theta}\left(\left(\pi_{\Sigma}^{\theta}\right)^{k}(\vec{\phi})\right) & =\sigma_{\Sigma}^{\theta}\left(\vec{\phi} / \theta_{\Sigma}\right) \\
& \left.=\sigma_{\Sigma}(\vec{\phi}) / \theta_{\Sigma}\right) \\
& =\pi_{\Sigma}^{\theta}\left(\sigma_{\Sigma}(\vec{\phi})\right) .
\end{aligned}
$$

Thus $\left\langle I_{\text {Sign }}, \pi^{\theta}\right\rangle$ is also an $\left(N, N^{\theta}\right)$-morphism and, therefore, an $\left(N, N^{\theta}\right)$-bilogical morphism from $\mathcal{I}$ to $\mathcal{I} / \theta$.

The surjective singleton interpretation (or $\left(N, N^{\theta}\right)$-bilogical morphism, if $\theta$ is a logical $N$-congruence system) $\left\langle I_{\text {Sign }}, \pi^{\theta}\right\rangle: \mathcal{I} \rightarrow \mathcal{I} / \theta$ will be referred to as the canonical quotient interpretation or canonical projection of $\mathcal{I}$ onto $\mathcal{I} / \theta$.

Proposition 23 has as consequence the fact that the category of theories of $\mathcal{I}$ and that of the theories of its logical quotient $\mathcal{I} / \theta$ are isomorphic categories via the isomorphism $\Pi^{\theta}$ induced by the singleton interpretation $\left\langle I_{\text {Sign }}, \pi^{\theta}\right\rangle$.

Corollary 24. Let $\mathcal{I}=\left\langle\right.$ Sign, SEN, $\left.\left\{C_{\sum}\right\}_{\sum \in|\operatorname{Sign}|}\right\rangle$ be a r-institution. If $\theta$ is a logical equivalence system of $\mathcal{I}$, then $\Pi^{\theta}: \operatorname{Th}(\mathcal{I}) \rightarrow \operatorname{Th}(\mathcal{I} / \theta)$, defined by

$$
\Pi^{\theta}\left(\left\langle\sum, T\right\rangle\right)=\left\langle\Sigma, \pi_{\Sigma}^{\theta}(T)\right\rangle, \quad \text { for all }\left\langle\sum, T\right\rangle \in|\mathbf{T h}(\mathcal{I})|,
$$

and $\Pi^{\theta}(f)=f$, for all $f \in \operatorname{Mor}(\mathbf{S i g n})$, is an isomorphism between the categories of theories of $\mathcal{I}$ and of $\mathcal{I} / \theta$.

Proof. Follows by combining Proposition 23 with Theorem 20 .

Using Theorem 21, we also get the following: 
Corollary 25. Let $\mathcal{I}=\left\langle\right.$ Sign, $\left.\mathrm{SEN},\left\{C_{\sum}\right\}_{\sum \in|\operatorname{Sign}|}\right\rangle$ be a $\pi$-institution and $N$ be a category of natural transformations on SEN. Then, if $\theta$ a logical $N$-congruence system of $\mathcal{I}$, then $\widetilde{\Omega}^{N^{\theta}}(\mathcal{I} / \theta)=\widetilde{\Omega}^{N}(\mathcal{I}) / \theta$, i.e.,

$$
\widetilde{\Omega}_{\Sigma}^{N^{\theta}}(\mathcal{I} / \theta)=\widetilde{\Omega}_{\Sigma}^{N}(\mathcal{I}) / \theta_{\Sigma}, \quad \text { for all } \sum \in|\mathbf{S i g n}| .
$$

\section{Bilogical Morphisms and Logical Quotients}

Suppose that $\mathcal{I}=\left\langle\right.$ Sign, SEN, $\left.\left\{C_{\sum}\right\}_{\sum \in|\operatorname{Sign}|}\right\rangle$ and $\mathcal{I}=\left\langle\right.$ Sign' $^{\prime}, \mathrm{SEN}^{\prime}$, $\left.\left\{C_{\sum}^{\prime}\right\}_{\sum \in\left|\mathbf{S i g n}^{\prime}\right|}\right\rangle$ are two $\pi$-institutions and $\langle F, \alpha\rangle: \mathcal{I} \vdash^{s} \mathcal{I}^{\prime}$ is a singleton interpretation from $\mathcal{I}$ to $\mathcal{I}^{\prime}$. Define $\theta^{\langle F, \alpha\rangle}=\left\{\left\langle\sum, \theta_{\Sigma}^{\langle F, \alpha\rangle}\right\rangle: \Sigma \in|\mathbf{S i g n}|\right\}$ by setting, for all $\sum \in|\mathbf{S i g n}|$,

$$
\theta_{\Sigma}^{\langle F, \alpha\rangle}=\left\{\langle\phi, \psi\rangle \in \operatorname{SEN}(\Sigma)^{2}: \alpha_{\Sigma}(\phi)=\alpha_{\Sigma}(\psi)\right\} .
$$

Proposition 26. Let $\mathcal{I}=\left\langle\right.$ Sign, $\left.\mathrm{SEN},\left\{C_{\sum}\right\}_{\sum \in|\operatorname{Sign}|}\right\rangle, \mathcal{I}=\left\langle\right.$ Sign' $^{\prime}, \mathrm{SEN}^{\prime}$, $\left.\left\{C_{\sum}^{\prime}\right\}_{\sum \in\left|\mathbf{S i g n}^{\prime}\right|}\right\rangle$ be two $\pi$-institutions.

(1) Given a singleton interpretation $\langle F, \alpha\rangle: \mathcal{I} \vdash^{s} \mathcal{I}^{\prime}, \theta^{\langle F, \alpha\rangle}$ is a logical equivalence system of $\mathcal{I}$.

(2) If $N$ and $N^{\prime}$ are categories of natural transformations on SEN and $\mathrm{SEN}^{\prime}$, respectively, and $\langle F, \alpha\rangle: \mathcal{I} \vdash^{s} \mathcal{I}^{\prime}$ is a strong $\left(N, N^{\prime}\right)$-logical morphism, then $\theta^{\langle F, \alpha\rangle}$ is a logical $N$-congruence system of $\mathcal{I}$.

Proof. It is obvious from the definition that $\theta_{\Sigma}^{\langle F, \alpha\rangle}$ is an equivalence relation on $\operatorname{SEN}(\Sigma)$, for all $\sum \in|\operatorname{Sign}|$. Moreover, $\theta^{\langle F, \alpha\rangle}$ is an equivalence system on SEN, since, for all $\langle\phi, \psi\rangle \in \theta_{\Sigma}^{\langle F, \alpha\rangle}, f \in \operatorname{Sign}\left(\sum, \Sigma^{\prime}\right)$, we have $\alpha_{\Sigma}(\phi)=\alpha_{\Sigma}(\psi)$ whence $\operatorname{SEN}(f)\left(\alpha_{\Sigma}(\phi)\right)=\operatorname{SEN}(f)\left(\alpha_{\Sigma}(\psi)\right)$ and, therefore, 


$$
\alpha_{\Sigma^{\prime}}(\operatorname{SEN}(f)(\phi))=\alpha_{\Sigma^{\prime}}(\operatorname{SEN}(f)(\psi)),
$$

i.e., $\langle\operatorname{SEN}(f)(\phi), \operatorname{SEN}(f)(\psi)\rangle \in \theta_{\Sigma^{\prime}}^{\langle F, \alpha\rangle}$. It is a logical equivalence system, since, for all $\sum \in|\mathbf{S i g n}|, \phi, \psi \in \operatorname{SEN}(\Sigma)$, we have $\langle\phi, \psi\rangle \in \theta_{\Sigma^{\prime}}^{\langle F, \alpha\rangle}$ implies $\alpha_{\Sigma}(\phi)=\alpha_{\Sigma}(\psi)$, whence $C_{F(\Sigma)}^{\prime}\left(\alpha_{\Sigma}(\phi)\right)=C_{F(\Sigma)}^{\prime}\left(\alpha_{\Sigma}(\psi)\right)$ and, therefore, since $\langle F, \alpha\rangle$ is an interpretation, $C_{\Sigma}(\phi)=C_{\Sigma}(\psi)$.

For the second part, suppose that $N, N^{\prime}$ are categories of natural transformations on $\mathrm{SEN}, \mathrm{SEN}^{\prime}$, respectively, and $\langle F, \alpha\rangle: \mathcal{I} \vdash^{s} \mathcal{I}^{\prime}$ is a strong $\left(N, N^{\prime}\right)$-logical morphism. It suffices, taking into account, the first part, to show that $\theta^{\langle F, \alpha\rangle}$ is preserved by every natural transformation $\sigma: \mathrm{SEN}^{k} \rightarrow \mathrm{SEN}$ in $N$. We, in fact, have

$$
\begin{aligned}
& \langle\vec{\phi}, \vec{\psi}\rangle \in\left(\theta_{\Sigma^{\prime}}^{\langle F, \alpha\rangle}\right)^{k} \quad \text { iff } \quad \alpha_{\Sigma}\left(\phi_{i}\right)=\alpha_{\Sigma}\left(\psi_{i}\right) \text {, for all } i<k \text {, } \\
& \text { implies } \quad \sigma_{F(\Sigma)}^{\prime}\left(\alpha_{\Sigma}^{k}(\vec{\phi})\right)=\sigma_{F(\Sigma)}^{\prime}\left(\alpha_{\Sigma}^{k}(\vec{\psi})\right) \text {, for all } \sigma^{\prime} \text { in } N^{\prime}, \\
& \text { iff } \quad \alpha_{\Sigma}\left(\sigma_{\Sigma}(\vec{\phi})\right)=\alpha_{\Sigma}\left(\sigma_{\Sigma}(\vec{\psi})\right) \text {, for all } \sigma \text { in } N \text {, } \\
& \text { iff }\left\langle\sigma_{\Sigma}(\vec{\phi}), \sigma_{\Sigma}(\vec{\psi})\right\rangle \in \theta_{\Sigma}^{\langle F, \alpha\rangle} \text {, for all } \sigma \text { in } N \text {, }
\end{aligned}
$$

whence $\theta^{\langle F, \alpha\rangle}$ is a logical $N$-congruence system of $\mathcal{I}$.

The surjective singleton interpretation $\left\langle I_{\text {Sign }}, \pi^{\theta^{\langle F, \alpha\rangle}}\right\rangle$ will sometimes be denoted by $\left\langle I_{\text {Sign }}, \pi^{\langle F, \alpha\rangle}\right\rangle$. According to the second part of Propositions 26 and 23 , if $\langle F, \alpha\rangle: \mathcal{I} \vdash^{s} \mathcal{I}^{\prime}$ is a strong $\left(N, N^{\prime}\right)$-logical morphism, $\left\langle I_{\text {Sign }}, \pi^{\langle F, \alpha\rangle}\right\rangle: \mathcal{I} \vdash^{s} \mathcal{I} / \theta^{\langle F, \alpha\rangle}$ is an $\left\langle N, N^{\theta^{\langle F, \alpha\rangle}}\right\rangle$-bilogical morphism.

Theorem 27. Suppose $\mathcal{I}=\left\langle\right.$ Sign, SEN,$\left.\left\{C_{\sum}\right\}_{\sum \in|\operatorname{Sign}|}\right\rangle, \mathcal{I}^{\prime}=\left\langle\right.$ Sign' $^{\prime}, \mathrm{SEN}^{\prime}$, $\left.\left\{C_{\Sigma}^{\prime}\right\}_{\sum \in\left|\mathbf{S i g n}^{\prime}\right|}\right\rangle$, are two $\pi$-institutions. 
(1) If $\langle F, \alpha\rangle: \mathcal{I} \vdash^{s} \mathcal{I}^{\prime}$ is a singleton interpretation, then, there exists a unique singleton interpretation $\langle G, \beta\rangle: \mathcal{I} / \theta^{\langle F, \alpha\rangle} \vdash^{s} \mathcal{I}^{\prime}$, that makes the following triangle commute:

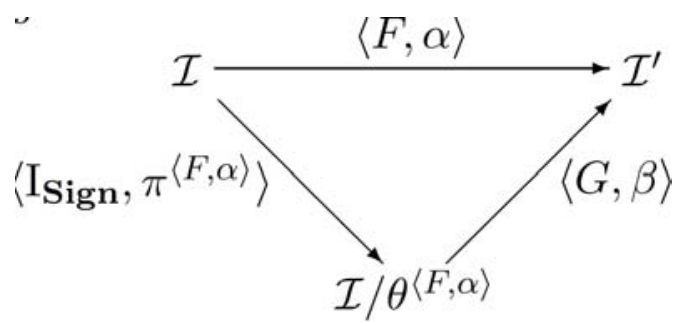

(2) If $N, N^{\prime}$ are categories of natural transformations on SEN, SEN', respectively, and $\langle F, \alpha\rangle: \mathcal{I} \vdash^{s} \mathcal{I}^{\prime}$ is a strong $\left(N, N^{\prime}\right)$-logical morphism, then the singleton interpretation $\langle G, \beta\rangle: \mathcal{I} / \theta^{\langle F, \alpha\rangle} \vdash^{s} \mathcal{I}^{\prime}$ of Part 1 is a strong $\left(N^{\theta^{\langle F, \alpha\rangle}}, N^{\prime}\right)$-logical morphism.

Proof. For simplicity of notation, denote inside this proof $\theta^{\langle F, \alpha\rangle}$ simply by $\theta$. Let $\langle G, \beta\rangle$ be given by $G=F$ and, for all $\sum \in|\mathbf{S i g n}|$, $\phi \in \operatorname{SEN}(\Sigma)$,

$$
\beta_{\Sigma}\left(\phi / \theta_{\Sigma}\right)=\alpha_{\Sigma}(\phi)
$$

The family of mappings $\beta$ is well defined, since $\langle\phi, \psi\rangle \in \theta_{\Sigma}$ implies $\alpha_{\Sigma}(\phi)=\alpha_{\Sigma}(\psi)$. It is a natural transformation $\beta: \mathrm{SEN}^{\theta} \rightarrow \mathrm{SEN}^{\prime} G$, since, for every $\Sigma_{1}, \Sigma_{2} \in|\operatorname{Sign}|, f \in \operatorname{Sign}\left(\Sigma_{1}, \Sigma_{2}\right)$ and $\phi \in \operatorname{SEN}\left(\Sigma_{1}\right)$,

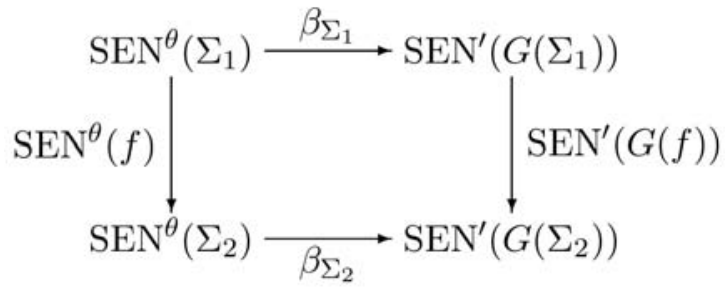




$$
\begin{aligned}
\operatorname{SEN}^{\prime}(G(f))\left(\beta_{\Sigma_{1}}\left(\phi / \theta_{\Sigma_{1}}\right)\right) & =\operatorname{SEN}^{\prime}(F(f))\left(\alpha_{\Sigma_{1}}(\phi)\right) \\
& =\alpha_{\Sigma_{2}}(\operatorname{SEN}(f)(\phi)) \\
& =\beta_{\Sigma_{2}}\left(\operatorname{SEN}(f)(\phi) / \theta_{\Sigma_{2}}\right) \\
& =\beta_{\Sigma_{2}}\left(\operatorname{SEN}^{\theta}(f)\left(\phi / \theta_{\Sigma_{1}}\right)\right) .
\end{aligned}
$$

The translation $\langle G, \beta\rangle$ is singleton, since $\langle F, \alpha\rangle$ is singleton, and it is an interpretation, since, for all $\Sigma \in|\operatorname{Sign}|, \Phi \bigcup\{\phi\} \subseteq \operatorname{SEN}(\Sigma)$,

$$
\begin{array}{lll}
\beta_{\Sigma}\left(\phi / \theta_{\Sigma}\right) \in C_{G(\Sigma)}^{\prime}\left(\beta_{\Sigma}\left(\Phi / \theta_{\Sigma}\right)\right) & \text { iff } & \alpha_{\Sigma}(\phi) \in C_{F(\Sigma)}^{\prime}\left(\alpha_{\Sigma}(\Phi)\right) \\
& \text { iff } \quad \phi \in C_{\Sigma}(\Phi) \\
& \text { iff } & \phi / \theta_{\Sigma} \in C_{\Sigma}^{\theta}\left(\Phi / \theta_{\Sigma}\right) .
\end{array}
$$

Finally, $\beta_{\Sigma}\left(\pi_{\Sigma}^{\theta}(\phi)\right)=\beta_{\Sigma}\left(\phi / \theta_{\Sigma}\right)=\alpha_{\Sigma}(\phi)$, for all $\Sigma \in|\operatorname{Sign}|, \phi \in \operatorname{SEN}(\Sigma)$, which concludes the proof of the first part.

For the second part, observe that, for all $\sum \in|\operatorname{Sign}|, \tau: \operatorname{SEN}^{k} \rightarrow$ SEN in $N$, we have commutativity of the following diagrams:
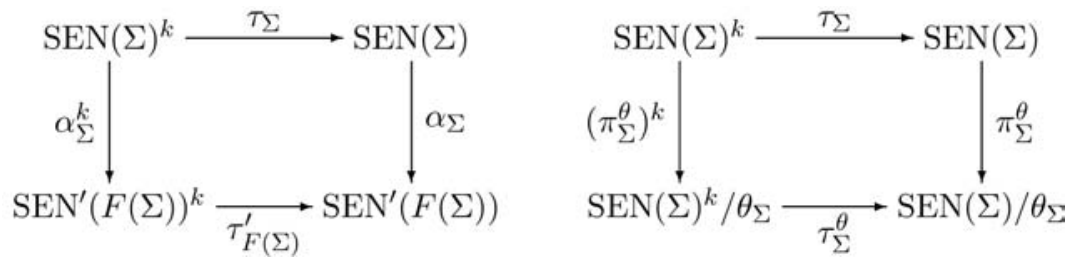

where by $\tau^{\prime}: \mathrm{SEN}^{\prime k} \rightarrow \mathrm{SEN}^{\prime}$ is denoted the natural transformation corresponding to $\tau$. By combining these, together with the definition of $\langle G, \beta\rangle$, we get

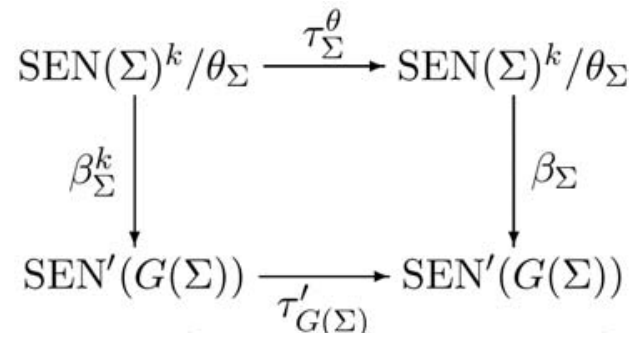




$$
\begin{aligned}
\tau_{G(\Sigma)}^{\prime}\left(\beta_{\Sigma}^{k}\left(\vec{\phi} / \theta_{\Sigma}\right)\right) & = & \tau_{G(\Sigma)}^{\prime}\left(\alpha \frac{k}{\Sigma}(\vec{\phi})\right) \\
& = & \alpha_{\Sigma}\left(\tau_{\Sigma}(\vec{\phi})\right) \\
& = & \beta_{\Sigma}\left(\tau_{\Sigma}(\vec{\phi}) / \theta_{\Sigma}\right) \\
& = & \beta_{\Sigma}\left(\tau_{\Sigma}^{\theta}\left(\vec{\phi} / \theta_{\Sigma}\right)\right) .
\end{aligned}
$$

Theorem 28. (1) Let $\mathcal{I}=\left\langle\right.$ Sign, SEN, $\left.\left\{C_{\sum}\right\}_{\sum \in|\operatorname{Sign}|}\right\rangle$ be a $\pi$-institution and $\theta, \theta^{\prime}$ be logical equivalence systems of $\mathcal{I}$ such that $\theta \leq \theta^{\prime}$. Then $\theta^{\prime} / \theta=\left\{\theta_{\Sigma}^{\prime} / \theta_{\Sigma}: \Sigma \in|\mathbf{S i g n}|\right\}$ is a logical equivalence system of $\mathcal{I} / \theta$ and, furthermore, $(\mathcal{I} / \theta) /\left(\theta^{\prime} / \theta\right) \cong^{s} \mathcal{I} / \theta^{\prime}$.

(2) Let $\mathcal{I}=\left\langle\right.$ Sign, SEN, $\left.\left\{C_{\sum}\right\}_{\sum \in \mid \text { Sign } \mid}\right\rangle$ be a r-institution, $N$ be a category of natural transformations on SEN, and $\theta, \theta^{\prime}$ be logical $N$ congruence systems of $\mathcal{I}$, such that $\theta \leq \theta^{\prime}$. Then $\theta^{\prime} / \theta$ is a logical $N^{\theta}$. congruence system of $\mathcal{I} / \theta$ and, furthermore, $(\mathcal{I} / \theta) /\left(\theta^{\prime} / \theta\right) \cong^{s} \mathcal{I} / \theta^{\prime}$.

Proof. It is clear that $\theta_{\Sigma}^{\prime} / \theta_{\Sigma}$ is an equivalence relation on $\operatorname{SEN}(\Sigma) / \theta_{\Sigma}$, for all $\Sigma \in|\operatorname{Sign}|$. The collection $\theta^{\prime} / \theta$ is an equivalence system on $\operatorname{SEN}^{\theta}$, since, for all $\Sigma_{1}, \Sigma_{2} \in|\operatorname{Sign}|, f \in \operatorname{Sign}\left(\Sigma_{1}, \Sigma_{2}\right)$ and all $\phi, \phi \in \operatorname{SEN}\left(\Sigma_{1}\right)$, if $\left\langle\phi / \theta_{\Sigma_{1}}, \psi / \theta_{\Sigma_{1}}\right\rangle \in \theta_{\Sigma_{1}}^{\prime} / \theta_{\Sigma_{1}}$, we get $\langle\phi, \psi\rangle \in \theta_{\Sigma_{1}}^{\prime}$, whence, since $\theta^{\prime}$ is an equivalence system, we get $\langle\operatorname{SEN}(f)(\phi), \operatorname{SEN}(f)$ $(\psi)\rangle \in \theta_{\Sigma_{2}}^{\prime}$, and, therefore,

$$
\left\langle\operatorname{SEN}(f)(\phi) / \theta_{\Sigma_{2}}, \operatorname{SEN}(f)(\psi) / \theta_{\Sigma_{2}}\right\rangle \in \theta_{\Sigma_{2}}^{\prime} / \theta_{\Sigma_{2}}
$$

i.e., $\left\langle\operatorname{SEN}^{\theta}(f)\left(\phi / \theta_{\Sigma_{1}}\right), \operatorname{SEN}^{\theta}(f)\left(\psi / \theta_{\Sigma_{1}}\right)\right\rangle \in \theta_{\Sigma_{2}}^{\prime} / \theta_{\Sigma_{2}}$. To show that $\theta^{\prime} / \theta$ is a logical equivalence system, suppose, $\sum \in|\operatorname{Sign}|$ and 
$\phi, \psi \in \operatorname{SEN}(\Sigma)$. Then, if $\left\langle\phi / \theta_{\Sigma}, \psi / \theta_{\Sigma}\right\rangle \in \theta_{\Sigma}^{\prime} / \theta_{\Sigma}$, we get $\langle\phi, \psi\rangle \in \theta_{\Sigma}^{\prime}$, whence, since $\theta^{\prime}$ is logical, $C_{\Sigma}(\phi)=C_{\Sigma}(\psi)$, i.e., $C_{\Sigma}^{\theta}\left(\phi / \theta_{\Sigma}\right)=C_{\Sigma}^{\theta}\left(\psi / \theta_{\Sigma}\right)$. Hence $\theta^{\prime} / \theta$ is logical.

Now define $\langle F, \alpha\rangle: \mathcal{I} / \theta^{\prime} \rightarrow(\mathcal{I} / \theta) /\left(\theta^{\prime} / \theta\right)$ by $F=I_{\text {Sign }}$, the identity functor on Sign, and, for all $\sum \in|\operatorname{Sign}|, \phi \in \operatorname{SEN}(\Sigma)$,

$$
\alpha_{\Sigma}\left(\phi / \theta_{\Sigma}^{\prime}\right)=\left(\phi / \theta_{\Sigma}\right) /\left(\theta_{\Sigma}^{\prime} / \theta_{\Sigma}\right) .
$$

Similarly, define $\langle G, \beta\rangle:(\mathcal{I} / \theta) /\left(\theta^{\prime} / \theta\right) \rightarrow \mathcal{I} / \theta^{\prime}$ by letting $G=I_{\text {Sign }}$ and, for all $\sum \in|\operatorname{Sign}|, \phi \in \operatorname{SEN}(\Sigma)$,

$$
\beta_{\Sigma}\left(\left(\phi / \theta_{\Sigma}\right) /\left(\theta_{\Sigma}^{\prime} / \theta_{\Sigma}\right)\right)=\phi / \theta_{\Sigma}^{\prime}
$$

It is not difficult to check that $\langle F, \alpha\rangle$ and $\langle G, \beta\rangle$ are surjective singleton interpretations and they are inverse of each other in the sense that

$$
\beta_{\Sigma}\left(\alpha_{\Sigma}\left(\phi / \theta_{\Sigma}^{\prime}\right)\right)=\phi / \theta_{\Sigma}^{\prime}
$$

and, similarly, $\alpha_{\Sigma}\left(\beta_{\Sigma}\left(\left(\phi / \theta_{\Sigma}\right) /\left(\theta_{\Sigma}^{\prime} / \theta_{\Sigma}\right)\right)\right)=\left(\phi / \theta_{\Sigma}\right) /\left(\theta_{\Sigma}^{\prime} / \theta_{\Sigma}\right)$, for all $\sum \in|\operatorname{Sign}|, \phi \in \operatorname{SEN}(\Sigma)$.

Finally, for the second part, it suffices to show that, if $N$ a category of natural transformations on $\mathrm{SEN}$ and $\theta, \theta^{\prime}$ are logical $N$-congruence systems of $\mathcal{I}$, such that $\theta \leq \theta^{\prime}$, then $\theta^{\prime} / \theta$ is a logical $N^{\theta}$-congruence system of $\mathcal{I} / \theta,\langle F, \alpha\rangle$ is an $\left(N^{\theta^{\theta^{\prime} / \theta}}, N^{\theta}\right)$-bilogical morphism and $\langle G, \beta\rangle$ is an $\left(N^{\theta^{\prime}}, N^{\theta^{\theta^{\prime} / \theta}}\right)$-bilogical morphism.

To this end, suppose that $\sum \in|\operatorname{Sign}|$ and $\phi, \phi \in \operatorname{SEN}(\Sigma)$. Then we have 


$$
\begin{array}{ll}
\left\langle\vec{\phi} / \theta_{\Sigma}, \vec{\psi} / \theta_{\Sigma}\right\rangle \in\left(\theta_{\Sigma}^{\prime} / \theta_{\Sigma}\right)^{k} \\
\text { iff } \quad\langle\vec{\phi}, \vec{\psi}\rangle \in\left(\theta_{\Sigma}^{\prime}\right)^{k} \\
\text { implies } & \left\langle\sigma_{\Sigma}(\vec{\phi}), \sigma_{\Sigma}(\vec{\psi})\right\rangle \in \theta_{\Sigma}^{\prime} \text {, for all } \sigma \text { in } N, \\
\text { iff } & \left\langle\sigma_{\Sigma}(\vec{\phi}) / \theta_{\Sigma}, \sigma_{\Sigma}(\vec{\psi}) / \theta_{\Sigma}\right\rangle \in \theta_{\Sigma}^{\prime} / \theta_{\Sigma} \text {, for all } \sigma \text { in } N, \\
\text { iff } & \left\langle\sigma_{\Sigma}^{\theta}\left(\vec{\phi} / \theta_{\Sigma}\right), \sigma_{\Sigma}^{\theta}\left(\vec{\psi} / \theta_{\Sigma}\right)\right\rangle \in \theta_{\Sigma}^{\prime} / \theta_{\Sigma}, \text { for all } \sigma^{\theta} \text { in } N^{\theta},
\end{array}
$$

whence $\theta^{\prime} / \theta$ is an $N^{\theta}$-logical congruence system of $\mathcal{I} / \theta$.

Finally, given $\sum \in|\operatorname{Sign}|, \vec{\phi} \in \operatorname{SEN}\left(\sum\right)^{k}$ and $\sigma: \operatorname{SEN}^{k} \rightarrow \operatorname{SEN}$ in $N$,

$$
\begin{aligned}
& \left(\left(\operatorname{SEN}(\Sigma) / \theta_{\Sigma}\right) /\left(\theta_{\Sigma}^{\prime} / \theta_{\Sigma}\right)\right)^{k} \stackrel{\sigma_{\Sigma}^{\theta^{\theta^{\prime} / \theta}}}{\longrightarrow}\left(\operatorname{SEN}(\Sigma) / \theta_{\Sigma}\right) /\left(\theta_{\Sigma}^{\prime} / \theta_{\Sigma}\right) \\
& \left.\operatorname{SEN}(\Sigma) / \theta_{\Sigma}^{\prime}\right)^{k} \longrightarrow \operatorname{SEN}(\Sigma) / \theta_{\Sigma}^{\prime} \\
& \alpha_{\Sigma}\left(\sigma_{\Sigma}^{\theta^{\theta^{\prime} / \theta}}\left(\left(\vec{\phi} / \theta_{\Sigma}\right) /\left(\theta_{\Sigma}^{\prime} / \theta_{\Sigma}\right)\right)\right)=\quad \quad=\quad \alpha_{\Sigma}\left(\sigma_{\Sigma}^{\theta}\left(\vec{\phi} / \theta_{\Sigma}\right) /\left(\theta_{\Sigma}^{\prime} / \theta_{\Sigma}\right)\right) \\
& =\alpha_{\Sigma}\left(\left(\sigma_{\Sigma}(\vec{\phi}) / \theta_{\Sigma}\right) /\left(\theta_{\Sigma}^{\prime} / \theta_{\Sigma}\right)\right) \\
& =\sigma_{\Sigma}(\vec{\phi}) / \theta_{\Sigma}^{\prime} \\
& =\sigma_{\Sigma}^{\theta^{\prime}}\left(\vec{\phi} / \theta_{\Sigma}^{\prime}\right) \\
& =\sigma_{\Sigma}^{\theta^{\prime}}\left(\alpha_{\Sigma}^{k}\left(\left(\vec{\phi} / \theta_{\Sigma}^{\prime}\right) /\left(\theta_{\Sigma}^{\prime} / \theta_{\Sigma}\right)\right)\right) \text {, }
\end{aligned}
$$

and, similarly, for $\langle G, \beta\rangle$.

Theorem 28, combined with Theorem 21, yield as a consequence that the Tarski congruence systems of $\mathcal{I} / \theta^{\prime}$ and of $(\mathcal{I} / \theta) /\left(\theta^{\prime} / \theta\right)$ are in correspondence. 
Corollary 29. Let $\mathcal{I}=\left\langle\operatorname{Sign}, \mathrm{SEN},\left\{C_{\sum}\right\}_{\sum \in|\operatorname{Sign}|}\right\rangle$ be a $\pi$-institution, $N$ be a category of natural transformations on SEN, and $\theta, \theta^{\prime}$ be logical $N$-congruence systems of $\mathcal{I}$, such that $\theta \leq \theta^{\prime}$. Then,

$$
\widetilde{\Omega}^{N^{\theta^{\theta^{\prime}} / \theta}}\left((\mathcal{I} / \theta) /\left(\theta^{\prime} / \theta\right)\right)=\left(\widetilde{\Omega}^{N}(\mathcal{I}) / \theta\right) /\left(\theta^{\prime} / \theta\right) .
$$

Proof. Using (both the notation and the result of) Theorem 28, we get

$$
\begin{array}{rlrl}
\widetilde{\Omega}_{\Sigma}^{N^{\theta^{\theta^{\prime}} / \theta}}\left((\mathcal{I} / \theta) /\left(\theta^{\prime} / \theta\right)\right) & = & \alpha_{\Sigma}\left(\widetilde{\Omega}_{\Sigma}^{N^{\theta^{\prime}}}\left(\mathcal{I} / \theta^{\prime}\right)\right) & \text { (by Theorem 21) } \\
& =\alpha_{\Sigma}\left(\widetilde{\Omega}_{\Sigma}^{N}(\mathcal{I}) / \theta_{\Sigma}^{\prime}\right) & \text { (by Corollary 25) } \\
& =\left(\widetilde{\Omega}_{\Sigma}^{N}(\mathcal{I}) / \theta_{\Sigma}^{\prime}\right) /\left(\theta_{\Sigma}^{\prime} / \theta_{\Sigma}\right)\left(\text { by definition of } \alpha_{\Sigma}\right) .
\end{array}
$$

Now, taking into account Theorem 28, together with the fact that the Tarski congruence system of a $\pi$-institution is the greatest logical congruence system, and considering any category of natural transformations $N$ together with the Tarski $N$-congruence system $\widetilde{\Omega}^{N}(\mathcal{I})$ in place of $\theta^{\prime}$, we obtain the following analog of Proposition 1.13 of [11].

Proposition 30. Let $\mathcal{I}=\left\langle\right.$ Sign, SEN, $\left.\left\{C_{\sum}\right\}_{\sum \in|\operatorname{Sign}|}\right\rangle$ be a $\pi$-institution, $N$ be a category of natural transformations on SEN, and $\theta$ be a logical $N$-congruence system of $\mathcal{I}$. Then,

$$
(\mathcal{I} / \theta) /\left(\widetilde{\Omega}^{N}(\mathcal{I}) / \theta\right) \cong^{s} \mathcal{I} / \widetilde{\Omega}^{N}(\mathcal{I}) .
$$

Given a $\pi$-institution $\mathcal{I}=\left\langle\right.$ Sign, SEN, $\left.\left\{C_{\sum}\right\}_{\sum \in|\operatorname{Sign}|}\right\rangle$ and a category of natural transformations $N$ on SEN, $\mathcal{I}$ is said to be $N$-reduced if it has only one logical $N$-congruence system, i.e., when $\widetilde{\Omega}^{N}(\mathcal{I})=\Delta^{\mathrm{SEN}}$, where $\Delta_{\Sigma}^{\mathrm{SEN}}=\{\langle\phi, \phi\rangle: \phi \in \operatorname{SEN}(\Sigma)\}, \Sigma \in|\operatorname{Sign}|$. Moreover, given a $\pi$-institution 
$\mathcal{I}$, as above, and a category $N$ of natural transformations on SEN, set $\mathcal{I}^{N}=\mathcal{I} / \widetilde{\Omega}^{N}(\mathcal{I})$ and call $\mathcal{I}^{N}$ the $N$-reduct of $\mathcal{I}$. This notation replaces in the present context the notation $\mathcal{I}^{*}$, which would be suggested by analogy with [11], since there might be a need to make the category $N$ of natural transformations on SEN explicit.

Using this terminology and notation, Proposition 30 may be rephrased as following:

Proposition 31. Let $\mathcal{I}=\left\langle\right.$ Sign, SEN,$\left.\left\{C_{\sum}\right\}_{\sum \in|\operatorname{Sign}|}\right\rangle$ be a $\pi$-institution, $N$ be a category of natural transformations on $\mathrm{SEN}$, and $\theta$ be a logical $N$-congruence system of $\mathcal{I}$. Then $(\mathcal{I} / \theta)^{N^{\theta}} \cong^{s} \mathcal{I}^{N}$.

It is shown next that, if two $\pi$-institutions are related by an $\left(N, N^{\prime}\right)$-bilogical morphism, with an isomorphic functor component, then their $N$ and $N^{\prime}$-reducts, respectively, are isomorphic $\pi$-institutions. This is the analog of Proposition 1.14 of [11] regarding abstract logics.

Proposition 32. Let $\mathcal{I}=\left\langle\right.$ Sign, SEN,$\left.\left\{C_{\sum}\right\}_{\sum \in|\operatorname{Sign}|}\right\rangle, \mathcal{I}^{\prime}=\left\langle\right.$ Sign', $^{\prime} \mathrm{SEN}^{\prime}$, $\left.\left\{C_{\sum}^{\prime}\right\}_{\Sigma \in\left|\mathbf{S i g n}^{\prime}\right|}\right\rangle$ be two $\pi$-institutions and $N, N^{\prime}$ be categories of natural transformations on SEN, $\mathrm{SEN}^{\prime}$, respectively. If $\langle F, \alpha\rangle: \mathcal{I} \vdash^{s} \mathcal{I}^{\prime}$ is an $\left(N, N^{\prime}\right)$-bilogical morphism from $\mathcal{I}$ to $\mathcal{I}^{\prime}$, with $F: \mathbf{S i g n} \rightarrow \mathbf{S i g n}^{\prime}$ an isomorphism, then there exists an isomorphism $\langle F, \gamma\rangle: \mathcal{I}^{N} \cong^{s} \mathcal{I}^{N^{\prime}}$.

Proof. Let $G:$ Sign' $\rightarrow$ Sign denote the inverse functor of $F$. Define $\langle F, \gamma\rangle$ by setting, for all $\sum \in|\operatorname{Sign}|$ and all $\phi \in \operatorname{SEN}(\Sigma)$,

$$
\gamma_{\Sigma}\left(\phi / \widetilde{\Omega}_{\Sigma}^{N}(\mathcal{I})\right)=\alpha_{\Sigma}(\phi) / \widetilde{\Omega}_{F(\Sigma)}^{N^{\prime}}\left(\mathcal{I}^{\prime}\right), \quad \text { i.e., } \quad \gamma_{\Sigma}\left(\phi^{N}\right)=\alpha_{\Sigma}(\phi)^{N^{\prime}} .
$$

For all $\sum \in|\operatorname{Sign}|, \gamma_{\Sigma}$ is well-defined. This follows from Theorem 21. Moreover, $\gamma: \mathrm{SEN}^{N} \rightarrow \mathrm{SEN}^{N^{\prime}}$ is a natural transformation, since, for all $\Sigma_{1}, \Sigma_{2} \in|\operatorname{Sign}|, f \in \operatorname{Sign}\left(\Sigma_{1}, \Sigma_{2}\right)$ and all $\phi \in \operatorname{SEN}\left(\Sigma_{1}\right)$, 


$$
\begin{aligned}
\operatorname{SEN}^{N}\left(\Sigma_{1}\right) \stackrel{\gamma_{\Sigma_{1}}}{\longrightarrow} \operatorname{SEN}^{N}(f) \mid & \\
\operatorname{SEN}^{N}\left(\Sigma_{2}\right) \stackrel{N^{\prime}}{\gamma_{\Sigma_{2}}}\left(F\left(\Sigma_{1}\right)\right) & \operatorname{SEN}^{\prime N^{\prime}}\left(F\left(\Sigma_{2}\right)\right) \\
\operatorname{SEN}^{\prime N^{\prime}}(F(f))\left(\gamma_{\Sigma_{1}}\left(\phi^{N}\right)\right) & =\operatorname{SEN}^{\prime N^{\prime}}(F(f))\left(\alpha_{\Sigma_{1}}(\phi)^{N^{\prime}}\right) \\
& =\operatorname{SEN}^{\prime}(F(f))\left(\alpha_{\Sigma_{1}}(\phi)\right)^{N^{\prime}} \\
& =\alpha_{\Sigma_{2}}(\operatorname{SEN}(f)(\phi))^{N^{\prime}} \\
& =\gamma_{\Sigma_{2}}\left(\operatorname{SEN}(f)(\phi)^{N}\right) \\
& \gamma_{\Sigma_{2}}\left(\operatorname{SEN}(f)\left(\phi^{N}\right)\right) .
\end{aligned}
$$

It is clear that $\langle F, \gamma\rangle$ is surjective. Theorem 21 shows that it is also injective. Thus, it suffices to show that, for all $\Sigma \in|\operatorname{Sign}|$ and all $\Phi \cup\{\phi\}$ $\subseteq \operatorname{SEN}\left(\sum\right), \phi^{N} \in C_{\Sigma}^{N}\left(\Phi^{N}\right)$ iff $\gamma_{\Sigma}\left(\phi^{N}\right) \in C_{F(\Sigma)}^{\prime N^{\prime}}\left(\gamma_{\Sigma}\left(\Phi^{N}\right)\right)$. In fact, we have

$$
\begin{aligned}
& \phi^{N} \in C_{\Sigma}^{N}\left(\Phi^{N}\right) \quad \text { iff } \quad \phi \in \mathrm{C}_{\Sigma}(\Phi) \\
& \text { iff } \quad \alpha_{\Sigma}(\phi) \in C_{F(\Sigma)}^{\prime}\left(\alpha_{\Sigma}(\Phi)\right) \\
& \text { iff } \quad \alpha_{\Sigma}(\phi)^{N^{\prime}} \in C_{F(\Sigma)}^{\prime N^{\prime}}\left(\alpha_{\Sigma}(\Phi)^{N^{\prime}}\right) \\
& \text { iff } \quad \gamma_{\Sigma}\left(\phi^{N}\right) \in C_{F(\Sigma)}^{\prime N^{\prime}}\left(\gamma_{\Sigma}\left(\Phi^{N}\right)\right) \text {. }
\end{aligned}
$$

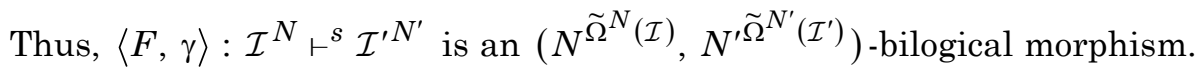
If one defines also $\langle G, \delta\rangle: \mathcal{I}^{N^{\prime}} \rightarrow \mathcal{I}^{N}$ by setting, for all $\Sigma \in\left|\mathbf{S i g n}^{\prime}\right|$ and all $\phi \in \operatorname{SEN}^{\prime}(\Sigma)$,

$$
\delta_{\Sigma}\left(\phi^{N^{\prime}}\right)=\gamma_{G(\Sigma)}^{-1}\left(\phi^{N^{\prime}}\right)
$$

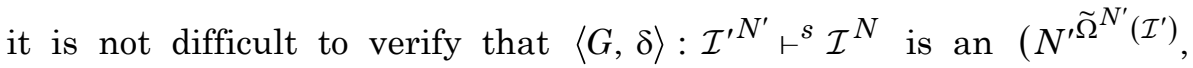
$\left.N^{\widetilde{\Omega}^{N}(\mathcal{I})}\right)$-bilogical morphism inverse to $\langle F, \gamma\rangle: \mathcal{I}^{N} \vdash^{s} \mathcal{I}^{\prime N^{\prime}}$. 


\section{Acknowledgements}

I acknowledge the constant support of Don Pigozzi. The work presented here started while I was writing my dissertation at Iowa State under his guidance. He has been with me ever since. Warm thanks to Charles Wells. I met him a few years later at Case Western Reserve and he has been a valuable source of inspiration and support. Thanks go also to Josep Maria Font and Ramon Jansana from Barcelona. They have contributed to the exploration of the Algebraic Logic landscape more than words can easily describe. Thanks, last but not least, to Janusz Czelakowski from Opole, whose contributions are also beyond easy description.

This paper would not have been possible without the pioneering work of Wim Blok. Blok passed away in 2003. He is missed by everyone working in this beautiful field.

If I can see as clearly, it is because you have been there before me and have illuminated the obscure path. I am grateful to each and every one of you.

\section{References}

[1] H. Albuquerque, J. M. Font and R. Jansana, Compatibility Operators in Abstract Algebraic Logic, preprint

[2] M. Barr and C. Wells, Category Theory for Computing Science, Third Edition, Les Publications CRM, Montréal, 1999.

[3] W. J. Blok and D. Pigozzi, Protoalgebraic logics, Studia Logica 45 (1986), 337-369.

[4] W. J. Blok and D. Pigozzi, Algebraizable logics, Memoirs of the American Mathematical Society 77 (1989), 396

[5] F. Borceux, Handbook of Categorical Algebra, Vol. I, Encyclopedia of Mathematics and its Applications, Cambridge University Press, 1994.

[6] D. J. Brown and R. Suszko, Abstract logics, Dissertationes Mathematicae 102 (1973), 9-42.

[7] J. Czelakowski, Equivalential logics I, II', Studia Logica 40 (1981), 227-236; 355-372.

[8] J. Czelakowski, Protoalgebraic Logics, Studia Logica Library 10, Kluwer, Dordrecht, 2001. 
[9] R. Diaconescu, Institution-Independent Model Theory, Studies in Universal Logic, Birkhäuser, Berlin, 2008.

[10] J. Fiadeiro and A. Sernadas, Structuring Theories on Consequence, in Recent Trends in Data Type Specification, Donald Sannella and Andrzej Tarlecki, Eds., Lecture Notes in Computer Science 332 (1988), 44-72.

[11] J. M. Font and R. Jansana, A General Algebraic Semantics for Sentential Logics, Lecture Notes in Logic, Vol. 7 (1996), Springer-Verlag, Berlin Heidelberg, 1996.

[12] J. M. Font, R. Jansana and D. Pigozzi, A survey of abstract algebraic logic, Studia Logica 74 (2003), 13-97.

[13] N. Galatos and C. Tsinakis, Equivalence of closure operators: an order-theoretic and categorical perspective, The Journal of Symbolic Logic 74 (2009), 780-810.

[14] N. Galatos and J. Gil-Férez, Modules over quantaloids: Applications to the isomorphism problem in algebraic logic and $\pi$-institutions, to appear in the Journal of Pure and Applied Algebra.

[15] J. Gil-Férez, Multi-term $\pi$-institutions and their equivalence, Mathematical Logic Quarterly 52 (2006), 505-526.

[16] J. A. Goguen and R. M. Burstall, Introducing Institutions, in Proceedings of the Logic of Programming Workshop, E. Clarke and D. Kozen, Eds., Lecture Notes in Computer Science 164 (1984), 221-256.

[17] J. A. Goguen and R. M. Burstall, Institutions: Abstract model theory for specification and programming, Journal of the Association for Computing Machinery 39(1992), 95-146.

[18] J. A. Goguen and R. Diaconescu, An introduction to category-based equational logic, AMAST 1995, Lecture Notes in Computer Science 936 (1995), 91-126.

[19] J. Goguen and G. Rosu, Institution morphisms, Formal Aspects of Computing 13 (2002), 274-307.

[20] S. Mac Lane, Category Theory for the Working Mathematician, Springer-Verlag, New York, 1971.

[21] G. Voutsadakis, Categorical abstract algebraic logic: Equivalent institutions, Studia Logica 74 (2003), 275-311.

[22] G. Voutsadakis, Categorical abstract algebraic logic: Algebraizable institutions, Applied Categorical Structures 10 (2002), 531-568.

[23] R. Wójcicki, Matrix approach in the methodology of sentential calculi, Studia Logica 32 (1973), 7-37. 